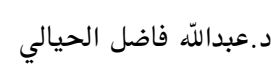
تطبيقات الذكاء الاقتصادي في التجارة..

\author{
تطبيقات الذكاء الاقتصادي في التجارة الالكترونية \\ في إطار الاقتصاد المبني على المعرفة

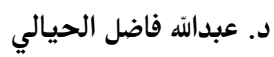 \\ مدرس / رئيس قسم الدراسات الاقتصادية والاجتماعية \\ مركز الدراسات الاقليمية/ جامعة الموصل
}

مستخلص البحث

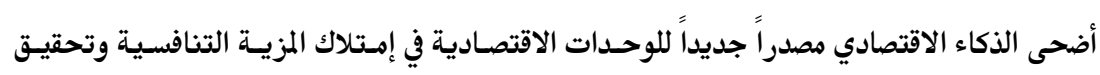

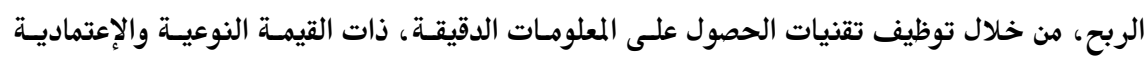

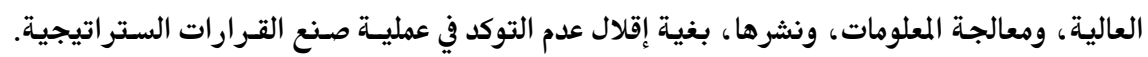

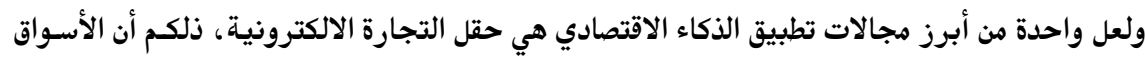

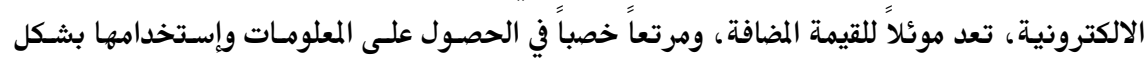
عقلاني، فضلاً عن معرفة نوع المنافسين، وماهية الخـدمات والمنتجـات الرقميـة والماديـة الجديـدة الـتي

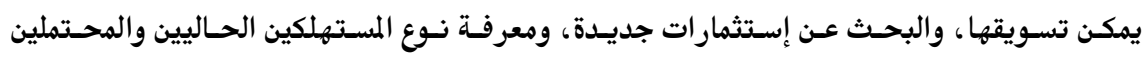

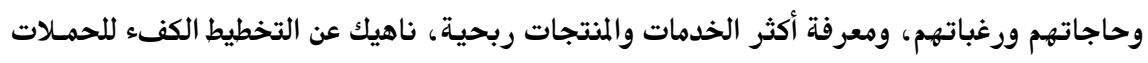

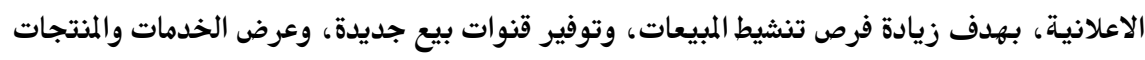

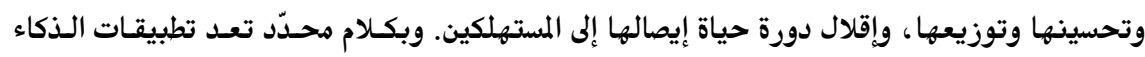

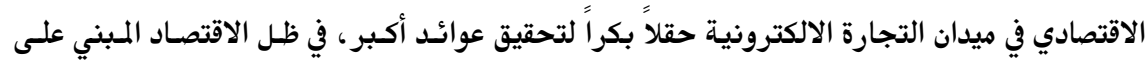

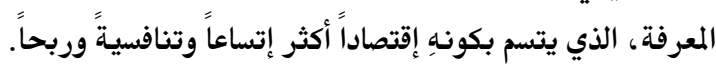


لقد وسّع الاقتصاد المبني على المعرفـة عـالم الاقتصـاد، ووجـدت الوحـدات الاقتصـادية

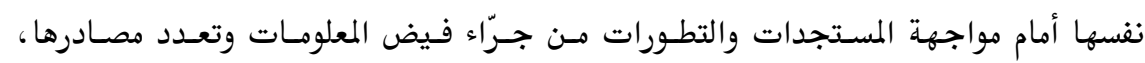

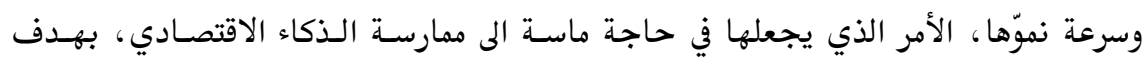

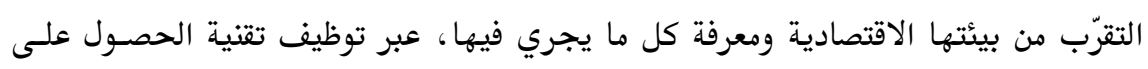

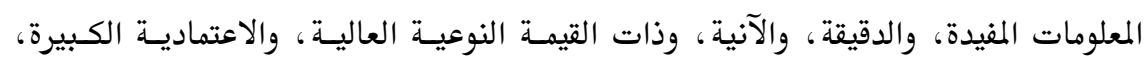

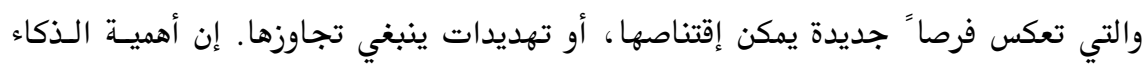

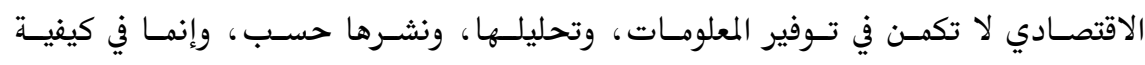

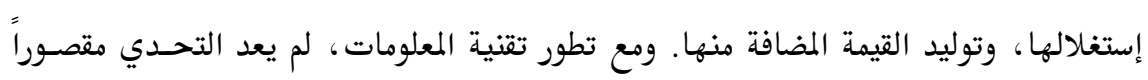

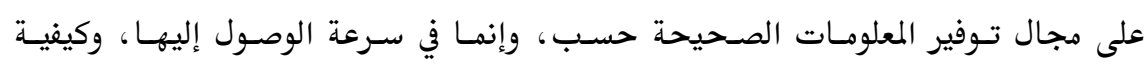

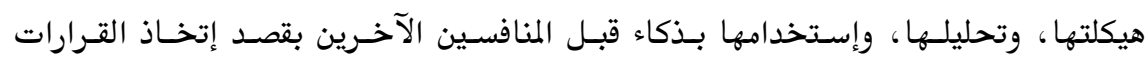

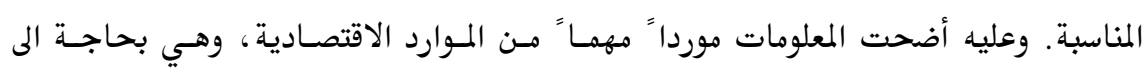

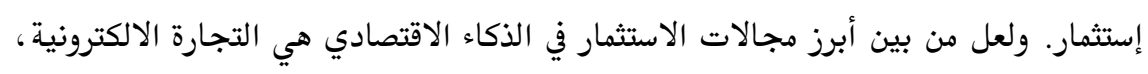

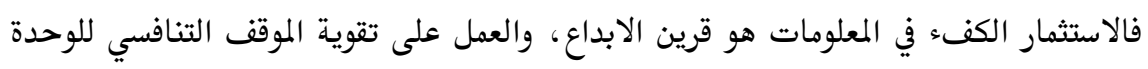

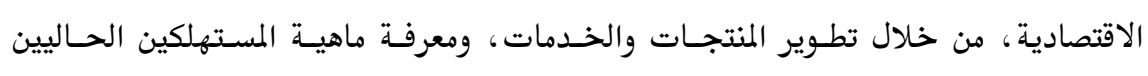

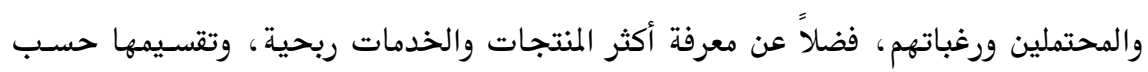
فئات معينة، وتوقعاتها المستقبلية، وتخطيط الحملات الدعائية لها.

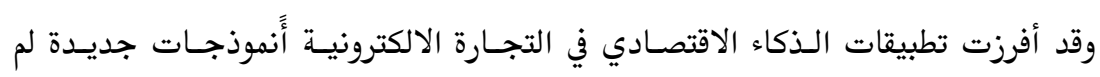

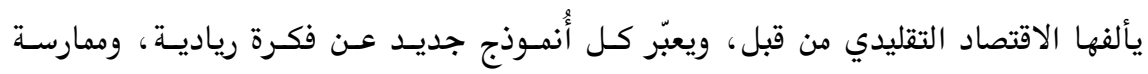

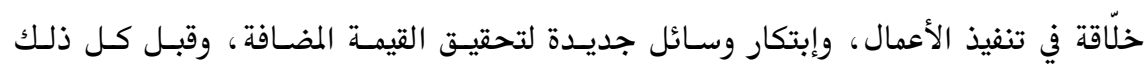

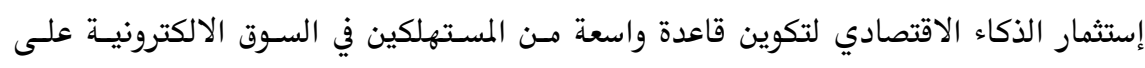

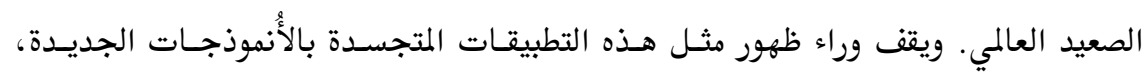

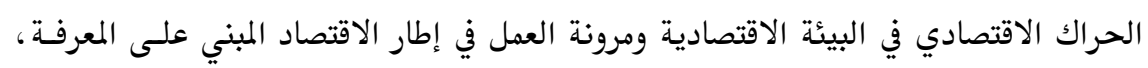

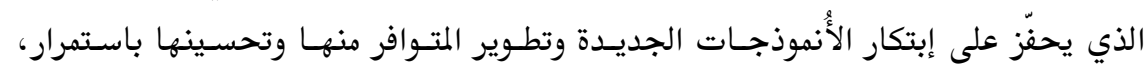

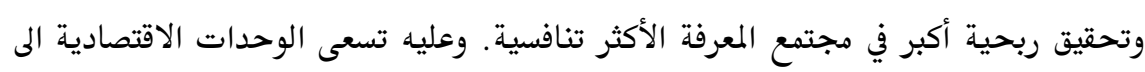

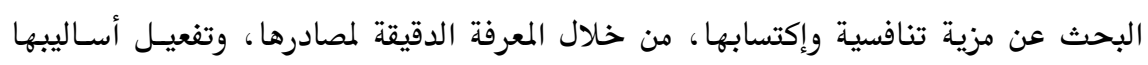




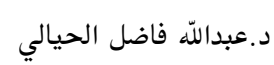
تطبيقات الذكاء الاقتصادي في التجارة..

بطريقة مبتكرة، ولا يكفي الحصول عليها حسب، وإنما تجديدها وتطويرها بشكل مستدام، ولن يتحقق ذلك إلا بتطبيق الذكاء الاقتصادي في بيئة إقتصادية شديدة التنافسية والدينامية.

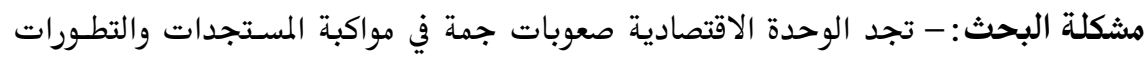

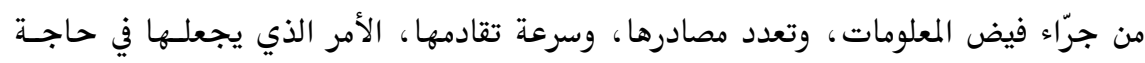

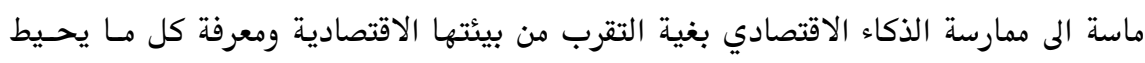

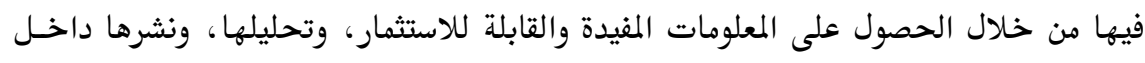

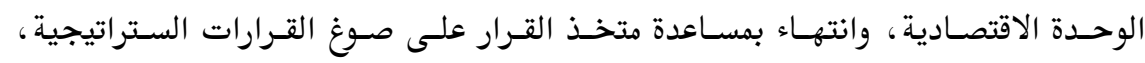
وإستثمار ذلك عبر تطبيقات عملية في ميدان التجارة الالكترونية. هدف البحث: يهدف البحث الى التعرّف على أهمية إستثمار الـذكاء الاقتصـادي وتطبيقاتسهـ

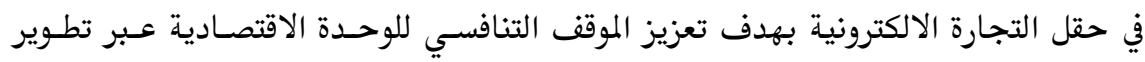

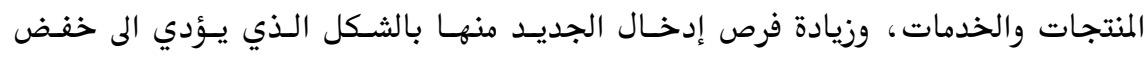
التكاليف وزيادة الأرباح. فرضية البحث: تؤدي ممارسة الذكاء الاقتصـادي في حقـل التجـارة الالكترونيـة الى تحقيـق

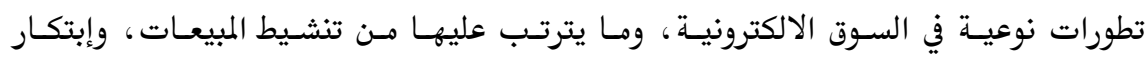
منتجات و خدمات جديدة، وإختزال دورة حياتها، وتعزيز الموقف التنافسي لها.

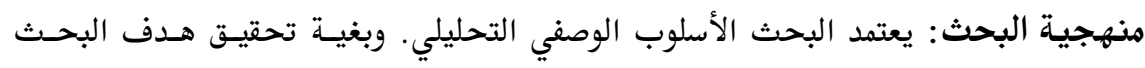

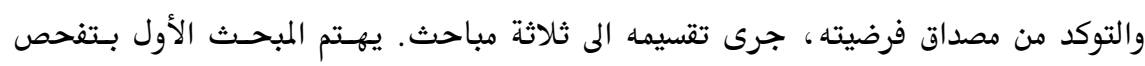

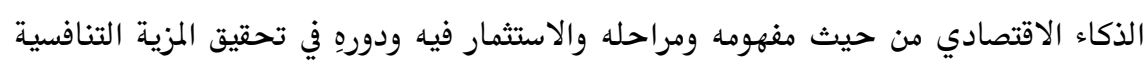
للوحدة الاقتصادية. ويتناول المبحث الثاني التجارة الالكترونية : ماهيتهـا وفئاتهـا وأهميتهـا

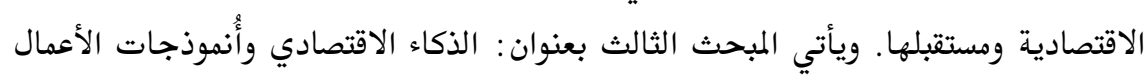

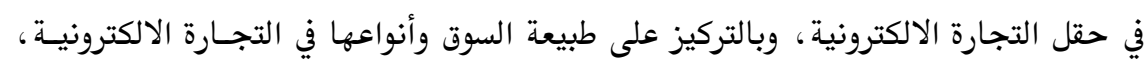

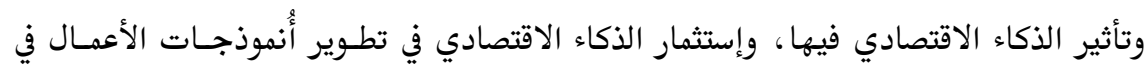
التجارة الالكترونية. ويختم البحث بتقديم الاستنتاجات والتوصيات. 
المبحث الاول: الذكاء الاقتصادي: مفهومسه ومراحلـه واستثماره ودوره في تحقيـق المزيـة

التنافسية

أولاًا / مفهوم الذكاء الاقتصادي

برز مفهوم الذكاء الاقتصـادي في منتصف سـتينات القـرن العشـرين، وتفضـل الأدبيـات

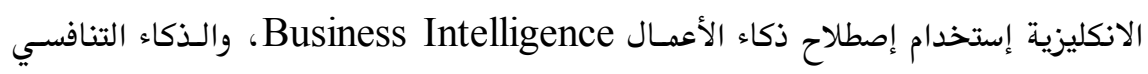

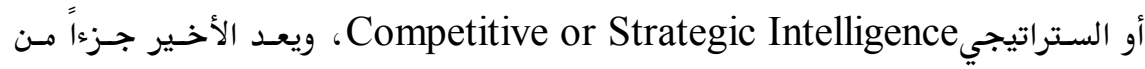

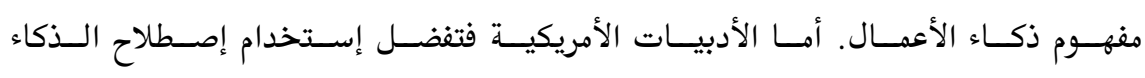

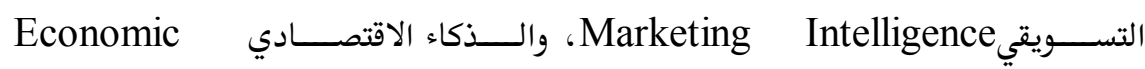

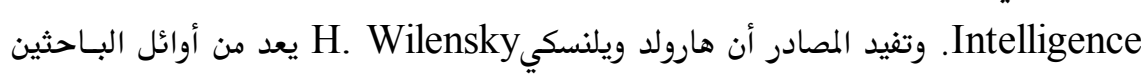

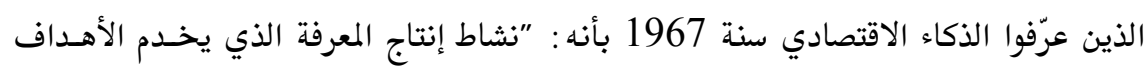

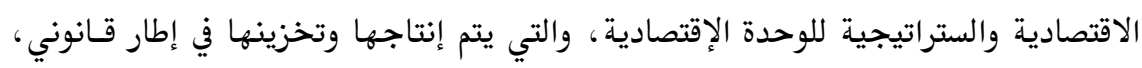
ومن مصادر مفتوحة "'(1).

وينصرف المعنى الاصطلاحي لمفهوم الـذكاء الاقتصـادي بحسب تعريـف بيرتيمـاكي وهـانولا Pirttimaki \& Hannula الاقتصادية من جمع، وتحليل، ونشر المعلومات المهمة الخاصـة بالنشـاطات الاقتصادية"(2).

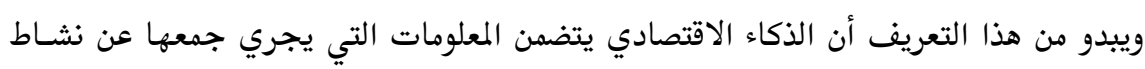

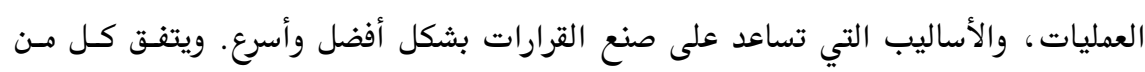

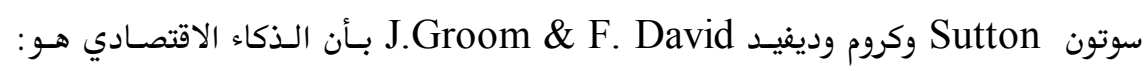

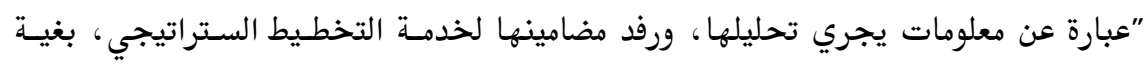

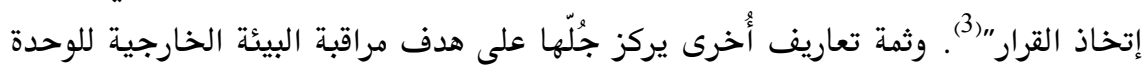

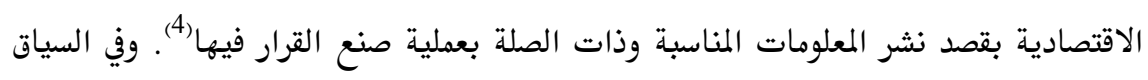
نفسـه ، يشـدّد هـامبلن Hamblin علسى عَدْْ الـذكاء الاقتصـادي : "عمليسة مراقبـة البيئسة

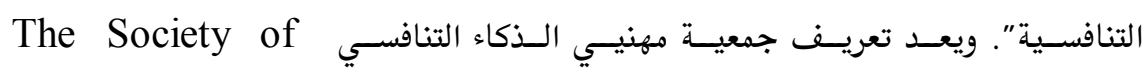
Competitive Intelligence professionals 


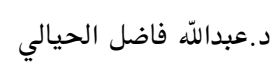
تطبيقات الذكاء الاقتصادي في التجارة..

أخلاقية تتضمن جمع، وتحليل، ونشر المعلومات الدقيقة، والمناسبة، والواضحة، والفعالـة،

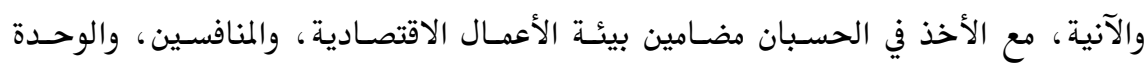

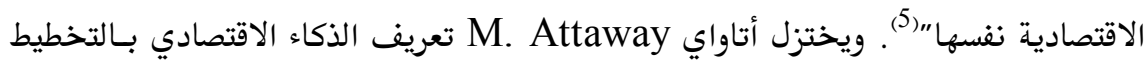

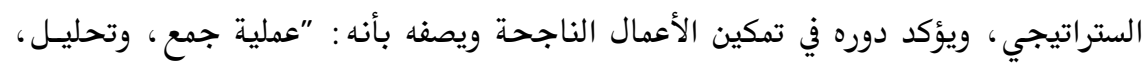

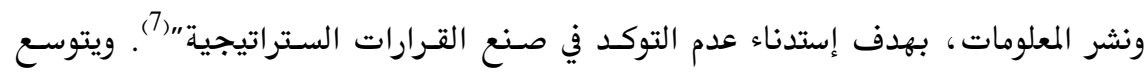

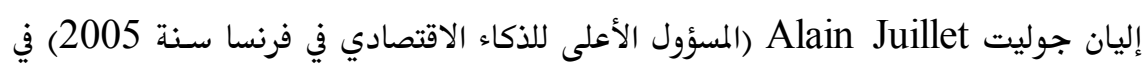

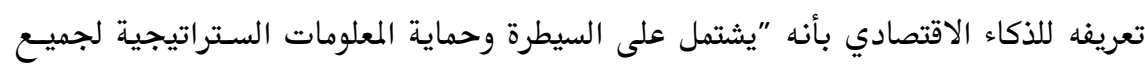

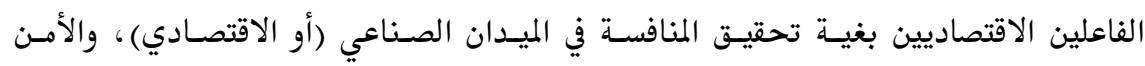

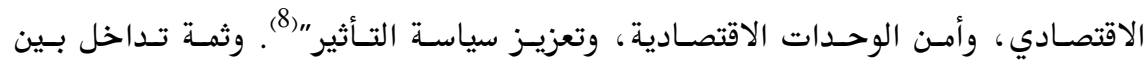

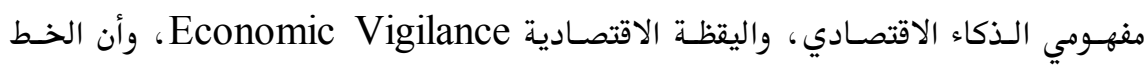

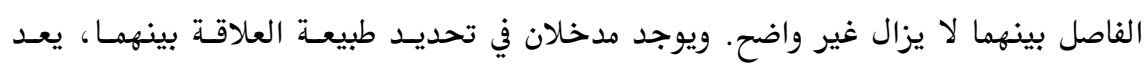

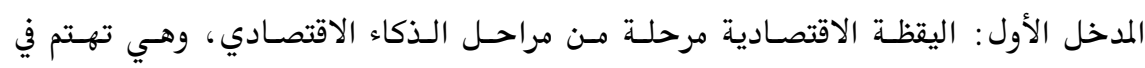

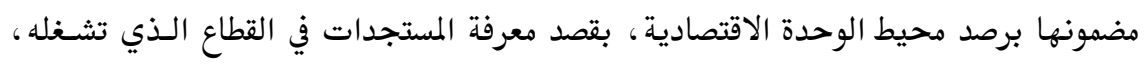

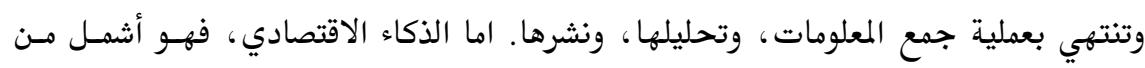

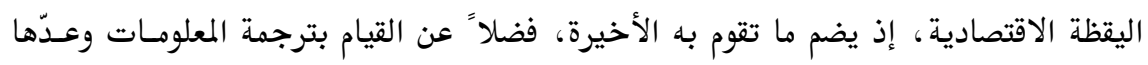

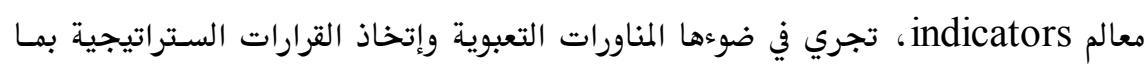

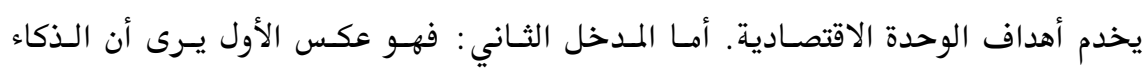

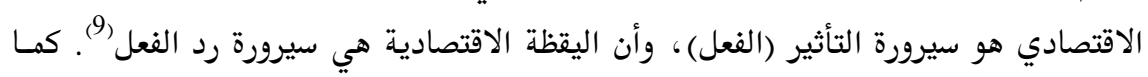

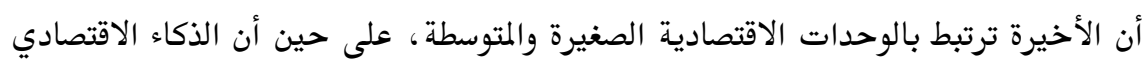

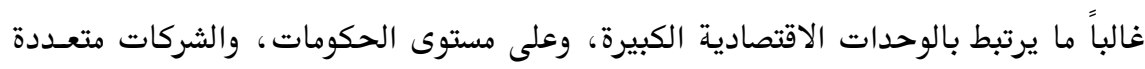
الجنسيات. وثمة أنواع عدة من اليقظة الاقتصادية نذكر أهمهاب(10): -

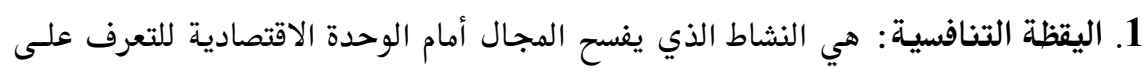

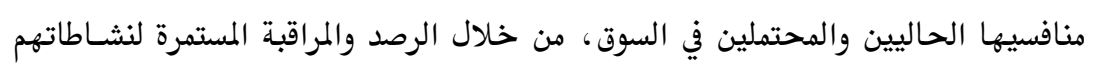

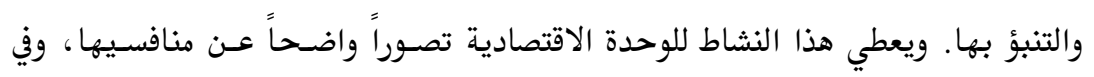


ضوء ذلك يتم وضع الخطط والستراتيجيات لإغتنام الفرص في السوق، وتجنب تهديداته الناجمة عن المنافسة.

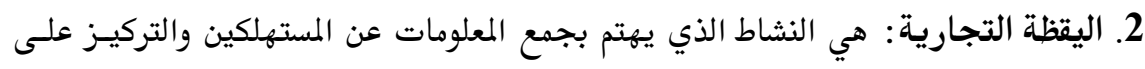

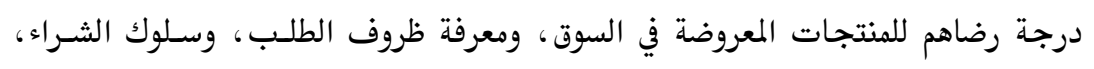

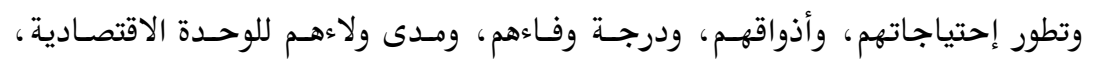

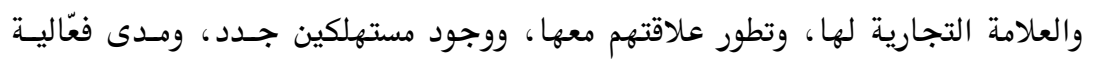

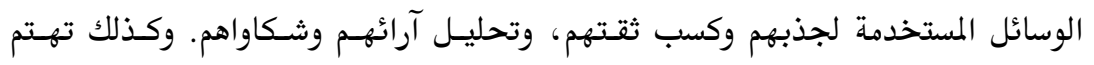

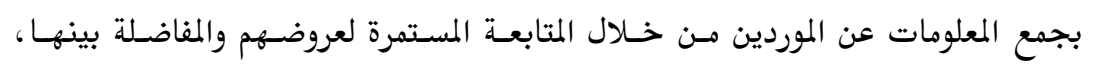

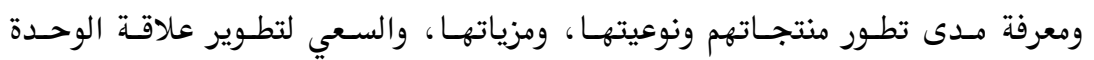

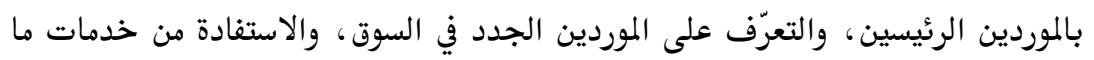
بعد البيع، والضمانات التي يتعهّدون بتقديمها.

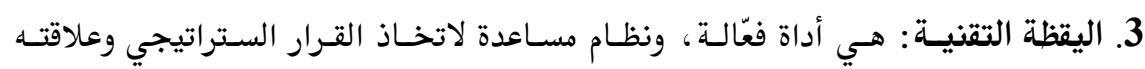

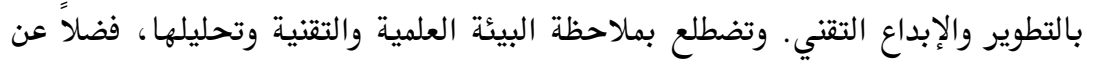

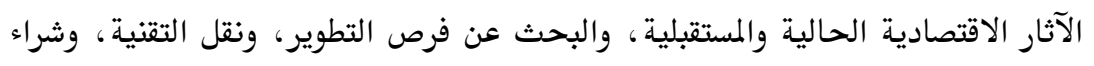

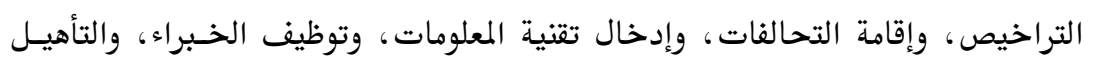
التقني.

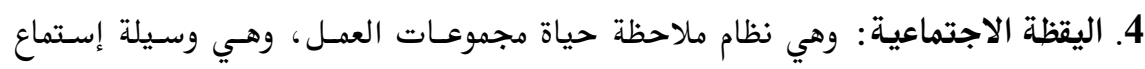

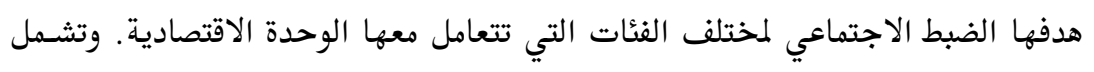

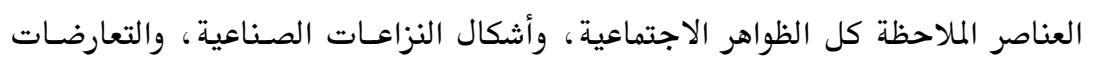

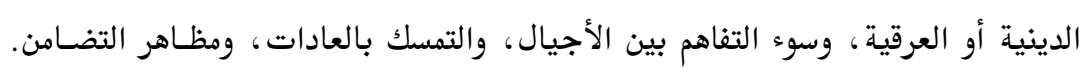

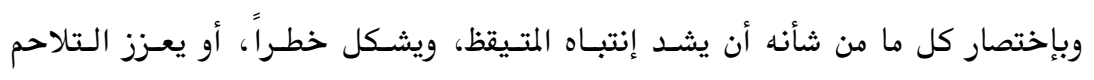
التنظيمي. 5. اليقظة البيئية: ينضوي تحت لواء هذا النوع كل ما يحيط ببيئة الوحدة الاقتصادية، أي

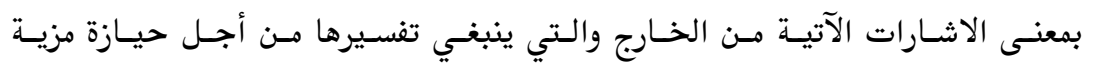


ستراتيجية لها. وتعمل على دراسة المتغيرات الاقتصادية والاجتماعيـة والثقافيـة للبيئسة الخارجية لها، لذا يطلق عليها البعض من الاقتصاديين باليقظة المجتمعية.

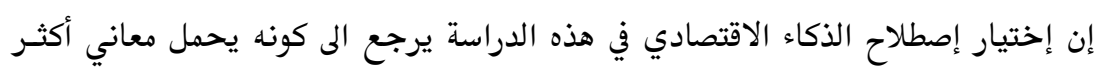

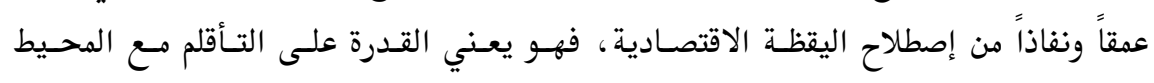

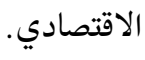

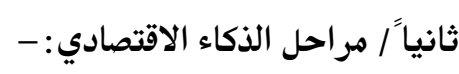

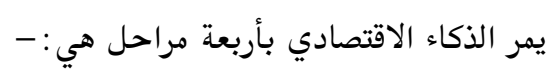
المرحلة الاولى: تحديد الحاجة للمعلومات

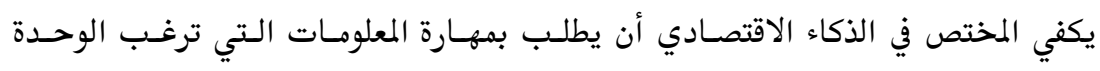

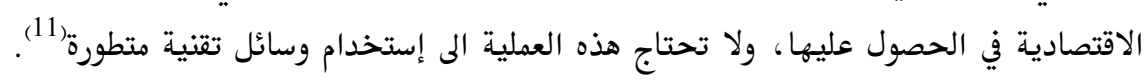

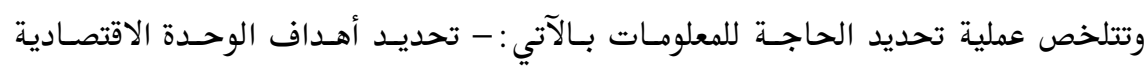

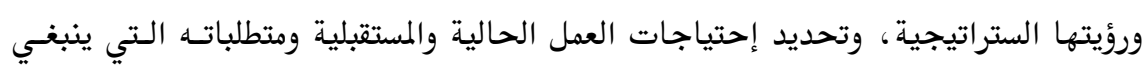
إضافتها أو تعديلها أو حذفها (12). المرحلة الثانية :- جمع المعلومات

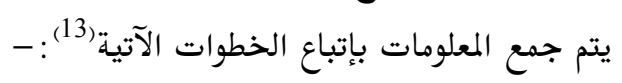

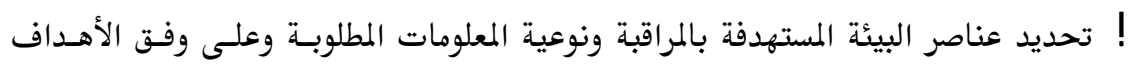
والحاجة المتوخاة من المعلومات.

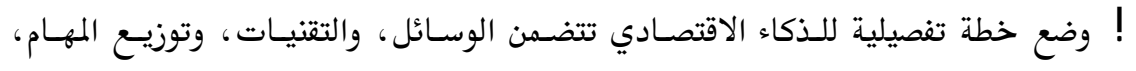
وخطة العمل، وميزانيته. - القيام بعملية جرد المصادر المشروعة التي يمكن الحصول منها على المعلومات المستهدفة.

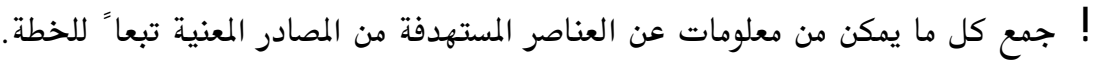

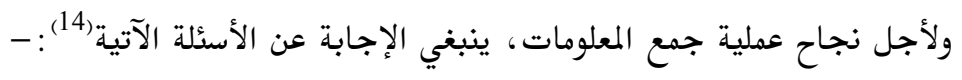


- هل أن المعلومات المتاحة حالياً من نظم المعلومـات المختلفـة صحيحة وكافيـة لفحصص

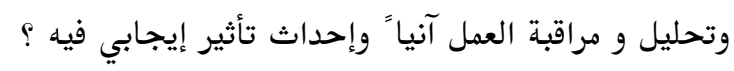

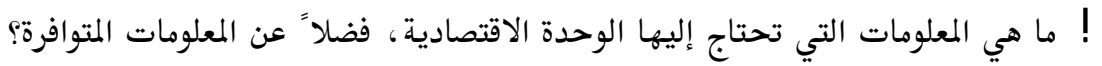

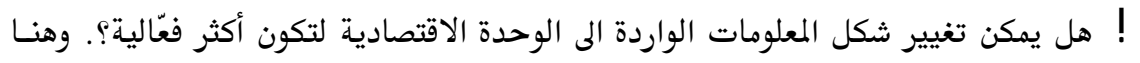
تبرز أهمية استخلاص البيانات، وتحويلها الى شكل مناسب، ومن ثم تحميلها الى مخزن

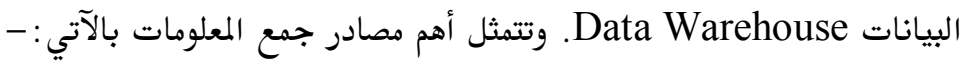

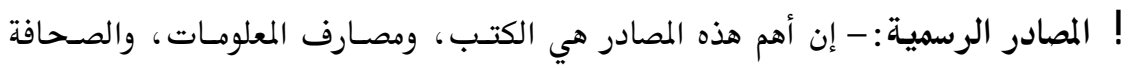

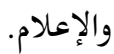
- المصـادر غـير الرسميـة: - إن أهـم مصــادر المعلوهـات غـير الرسميـة هـي المنافسـين، والموردين، والمصادر الداخلية للوحدة الاقتصادية. وثمة مصادر ثلاثة للحصول على المعلومات هي (15): -ـ الموردية.

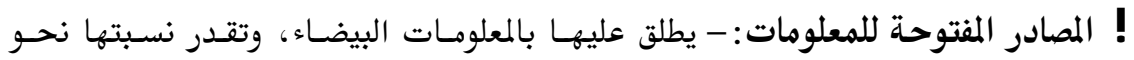
( المصاد (70\%) من إجمالي المصادر. - المصادر المغلقة للمعلومـات: - يطلـق عليهـا بالمعلومـات الرماديـة، وتقدر نسبتهـا نحـو (20\%) من إجمالي المصادر. - مصادر التجسس على المعلومات: - يطلق عليها بالمعلومات السوداء، وتقدر نسبتها نحسو (10\%) من إجمالي المصادر.

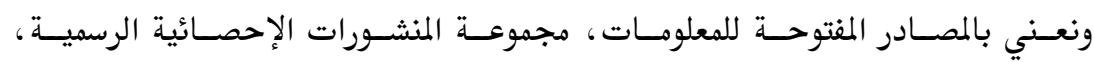

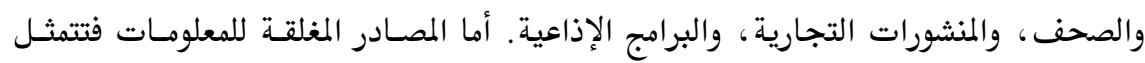

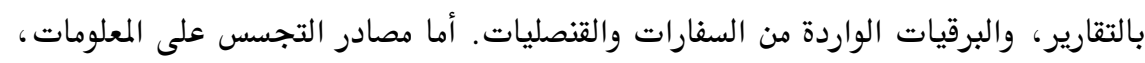

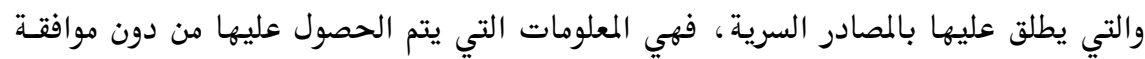

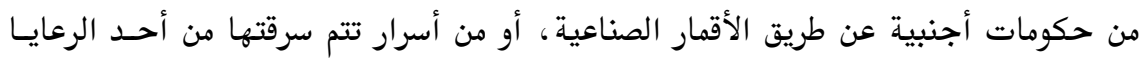

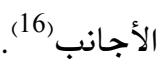




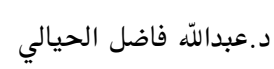
تطبيقات الذكاء الاقتصادي في التجارة..

يعد تحليل المعلومات أساس عملية الذكاء الاقتصادي، وتعتمد على قيمـة المعلومـات مسن

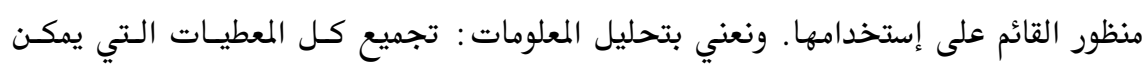
الحصول عليها من أجل تحليلها بشكل متجانس.

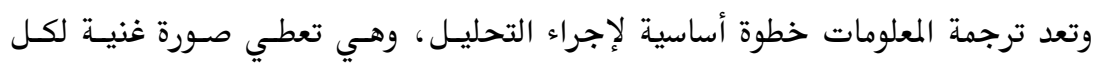
المعلومات التي تكون غير ظاهرة في سطور الوثائق(17).

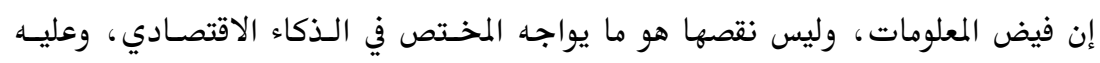
يتطلب منه القيام بالعمليات الآتية: - -

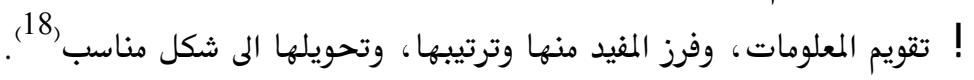
- تحليل المعلومات عبر تفسير دلالاتها الحالية، والتنبؤ بآثارها المستقبلية.

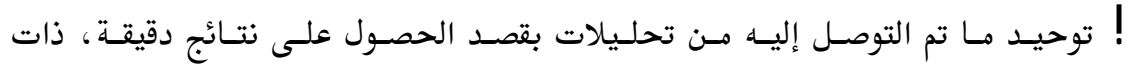
مصداقية (19).

المرحلة الرابعة: - نشر المعلومات وإتخاذ القرار

يجدر بالمختص في الذكاء الاقتصادي إعطاء قيمسة للمعلومـات بعـد تحليلـها، مـن خـلال

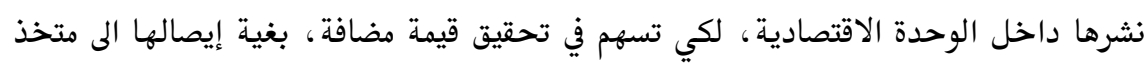

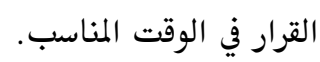

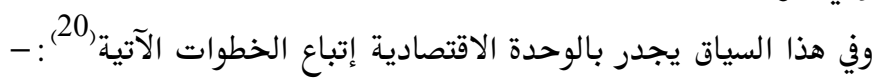

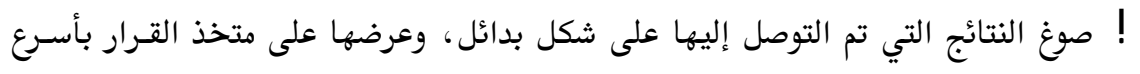
وقت لتفادي تقادم المعلومات. - دراسة متخذ القرار للبدائل المقترحة، و وإختيار أنسبها لتحقيق أهداف الوحدة الاقتصادية و أكثرها ملائمة لتطوير ستراتيجيات إبداعية ناجحة.

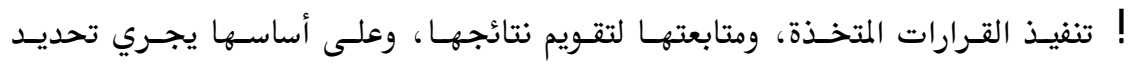

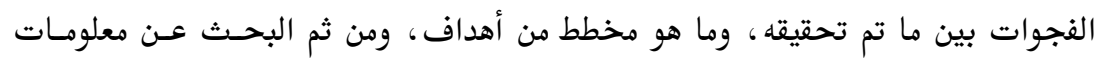
غنية تعمل على سد هذه الفجوات. 
ومـن الجـدير بالـذكر، أن عمليسة تحديـد الحاجـة للمعلومـات، وجمعهـا، وتحليلـها،

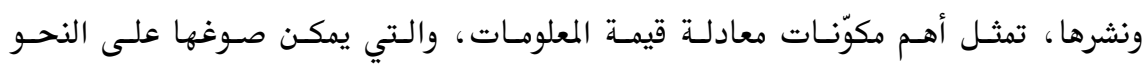

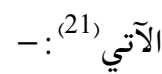

قيمة المعلومات = تحليل جيد للإحتياجات × صحة المصادر ونوعيتها × نوعية التحليل × النشر والتغذية الراجعة × الحماية.

\section{ثالثاً/ أهمية إستثمار الذكاء الاقتصادي}

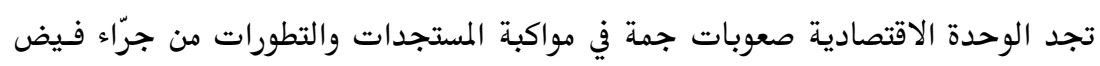

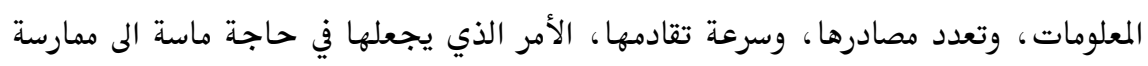

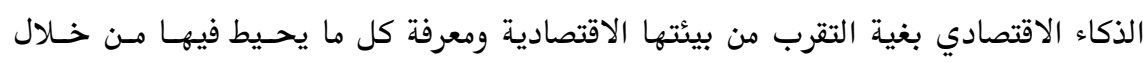

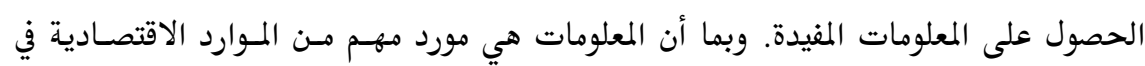

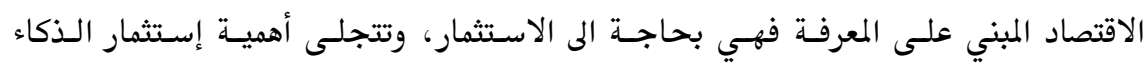

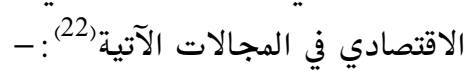

- تشجيع الإبداع: توصلت الدراسات الى وجود علاقة طردية بين الاستثمار في المعلومـات وبين الإبداع.

- تعزيز الموقف التنافسي للوحدة الاقتصادية: تمتلك الوحدات الاقتصـادية الناجحــ في

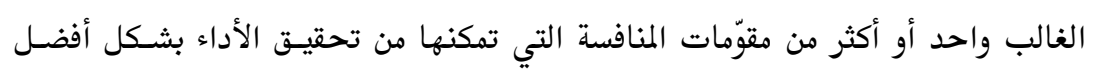

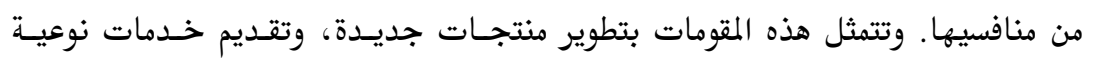

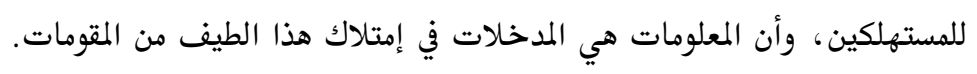

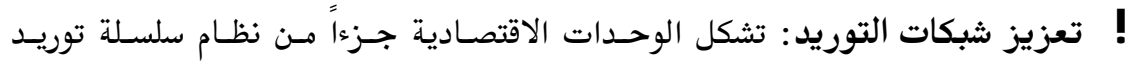

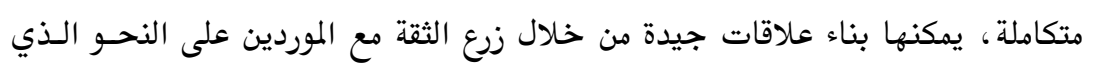

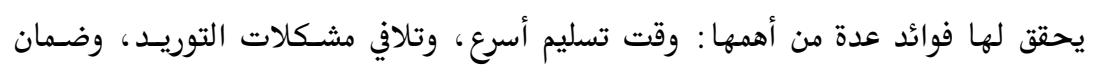
التوريد، ودعم فرص الحصول على الخصم، والتفضيل في المعاهلة والرعاية.

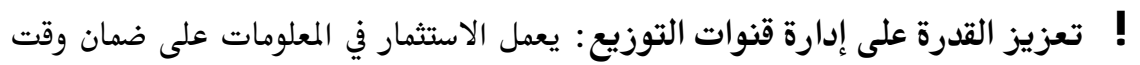

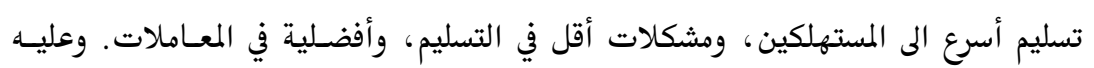


فأن الخبرة المكتسبة من تعلّم كيفية إستخدام نظم إدارة قنوات التوزيع يمكن أن يكـون قيداً مهماً في دخول المنافسين الجدد الى السوق.

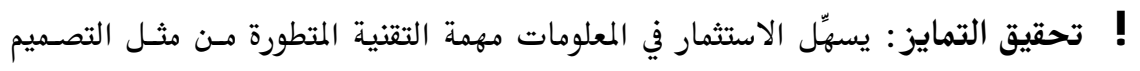

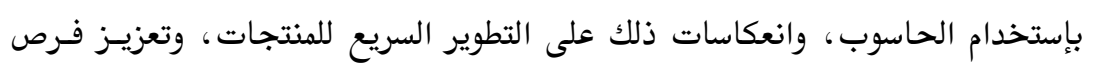

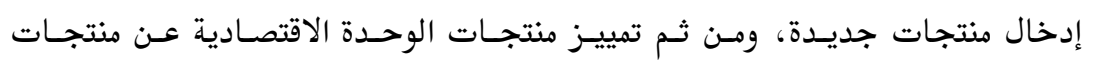

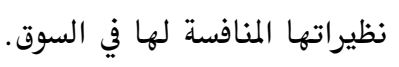

- تعزيز الاستقرار: تغيد الدراسات الى أن الاستثمار في المعلومات يسهم في تعزيز إستقرار

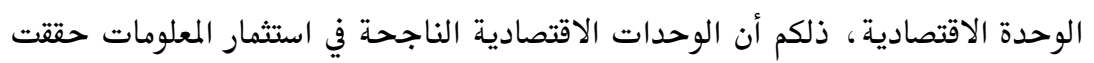

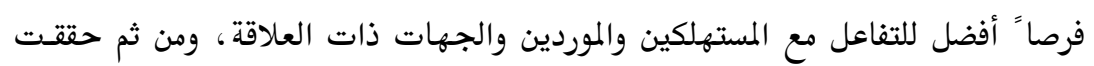
إستقراراً أفضل في أداء نشاطاتها.

\section{رابعاً/ الذكاء الاقتصادي مصدر جديد للمزية التنافسية}

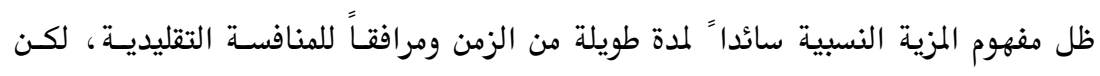

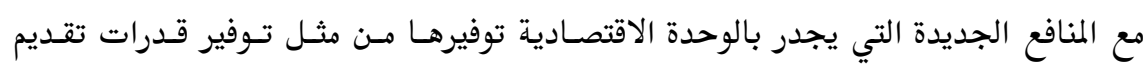

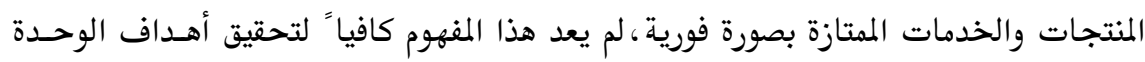

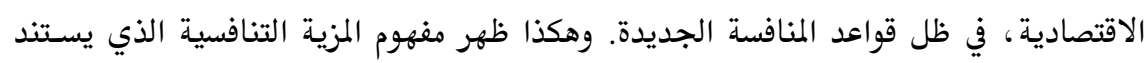

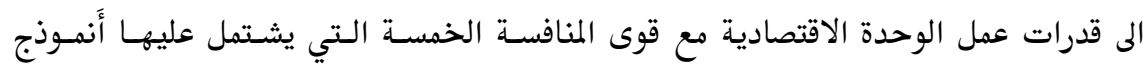

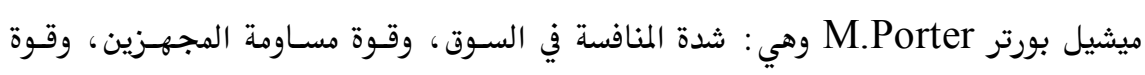

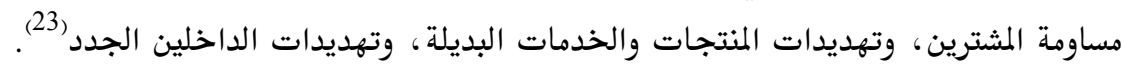

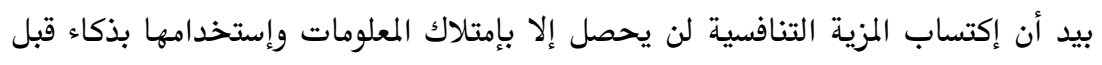

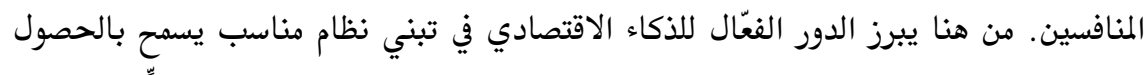

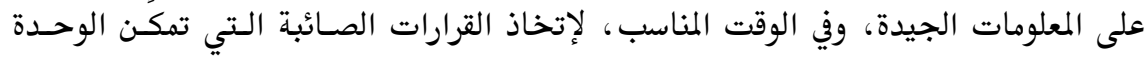

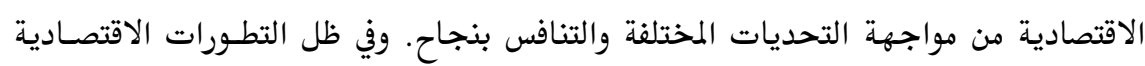

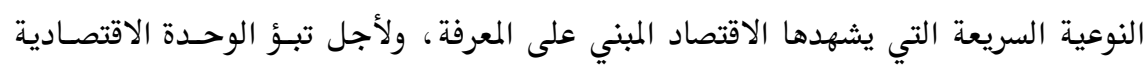

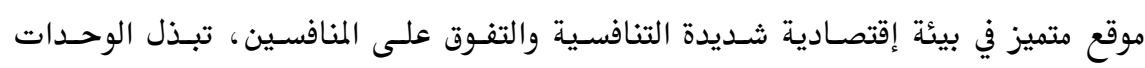


الاقتصادية جهودا" كبيرة في البحث عن مزية تنافسية حقيقية وإكتسابها من خـلال المعرفـة

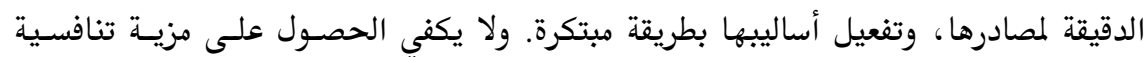

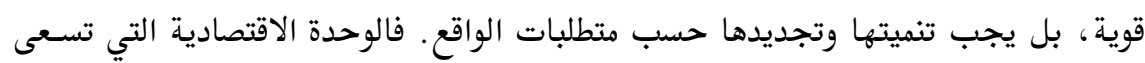

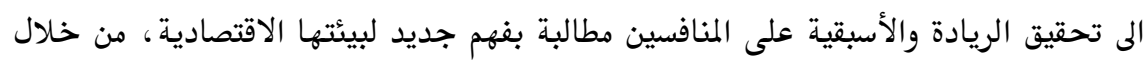

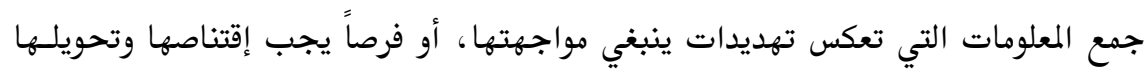

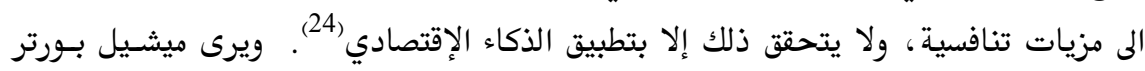
M.Porter Economic Rent بورتر فأن هناك نوعين من المزية التنافسية هما (25): -

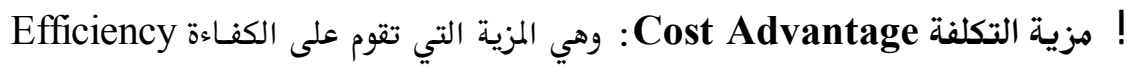

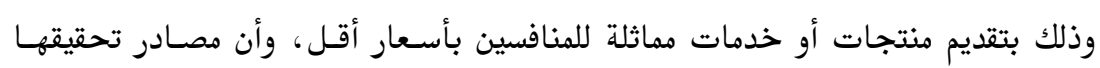

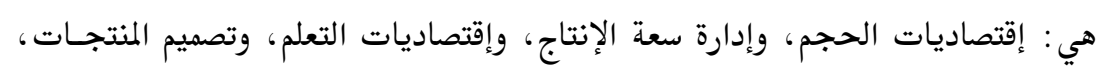
والتكلفة الدنيا للمدخلات، والكفاءة الإدارية، والتقنية العملية.

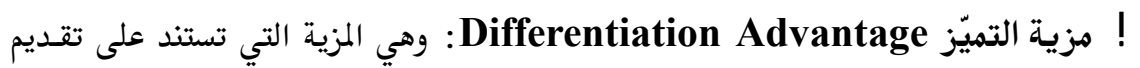

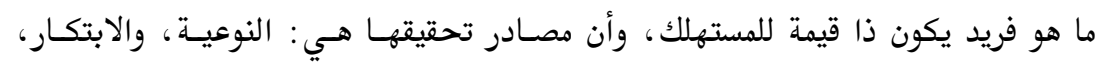

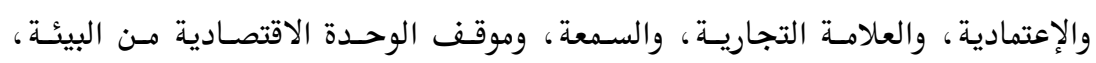

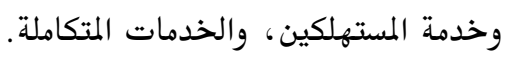

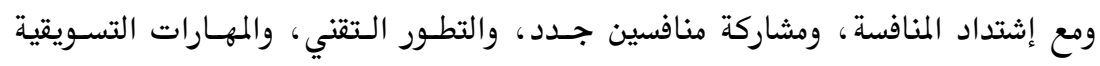

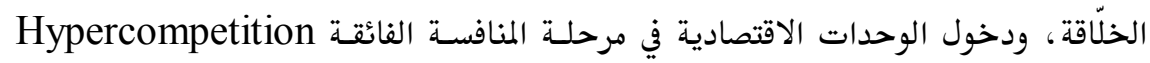

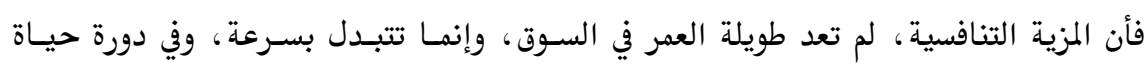

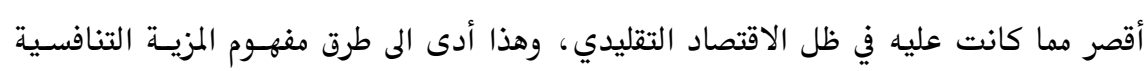

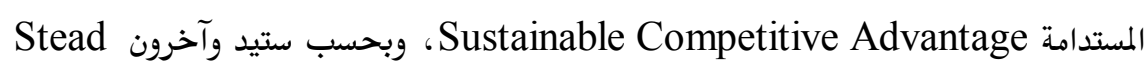
أن الاستدامة تتطلب طريقة جديدة للتفكير، كما تتطلب ذكـاءً إقتصـادياً (ستراتيجياً)

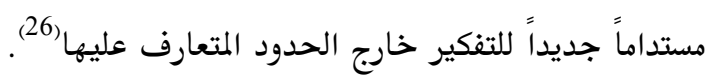




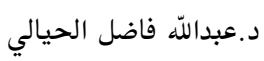

ويقال للوحدة الاقتصادية أن لديها مزية تنافسية مستدامة عندما تمتلك (بحسب جـاي

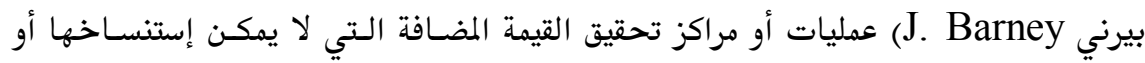

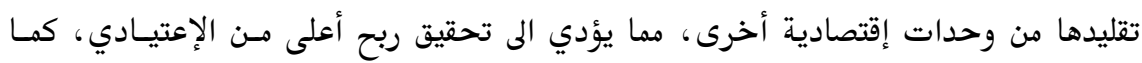

أنها تمثل مزية محسّنة وطويلة العمر للوحدة الاقتصادية في ظل المنافسة الفائقة (27).

المبحث الثاني :- التجارة الالكترونية: ماهيتها وفئاتها وأهميتها ومستقبلها. أولاً/ ماهية التجارة الالكترونية التحانية

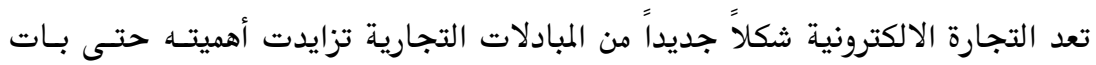

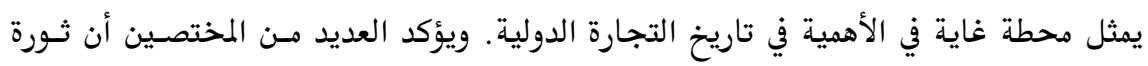

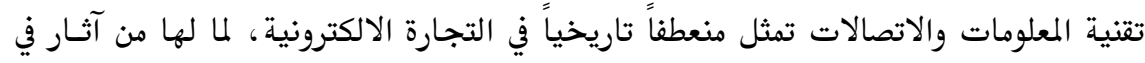

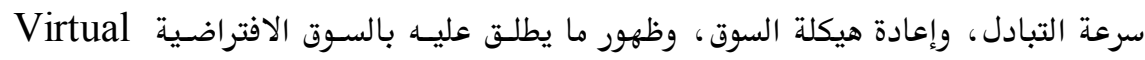
Marketspace

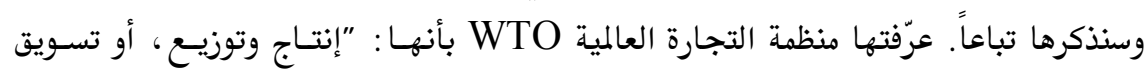

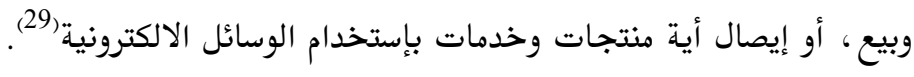

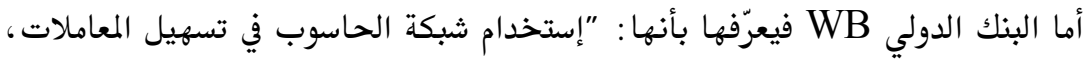

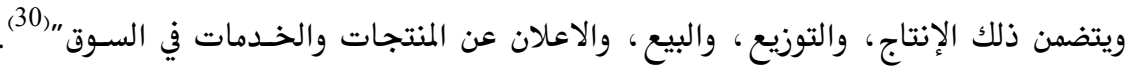

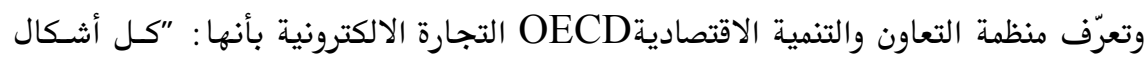

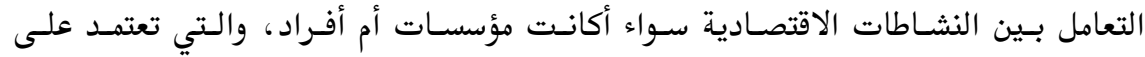

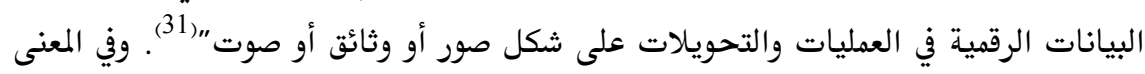

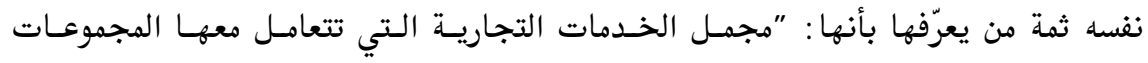

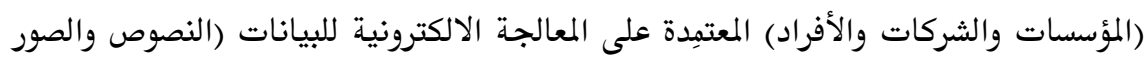

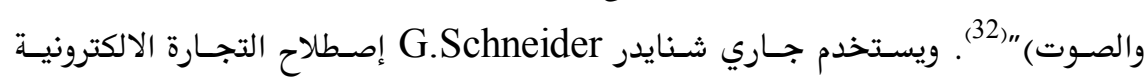
بمعناه الواسع كونها تشمل: "كل نشاطات الأعمال التي تنجز بإستخدام التبادل الالكترونسي

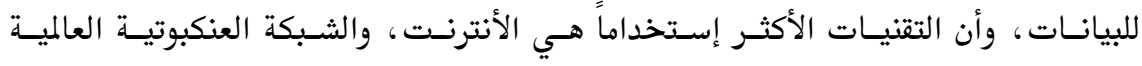

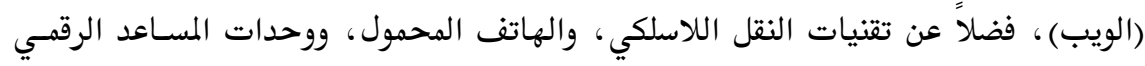


الشخصي Personal Digital Assistant (PDA)" (33). ويعـرّف كرينستاين وفينمسان Greenstein \& Feinman

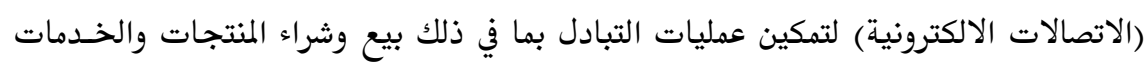

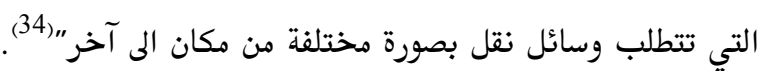

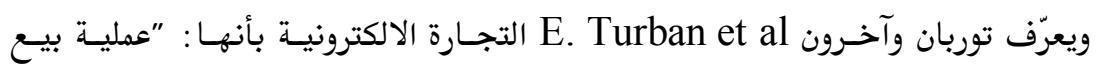
وشراء ومبادلة المنتجات والخدمات والمعلومات عبر شبكات الحساسوب" (35).

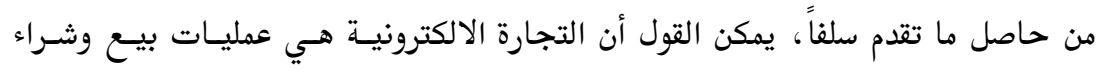

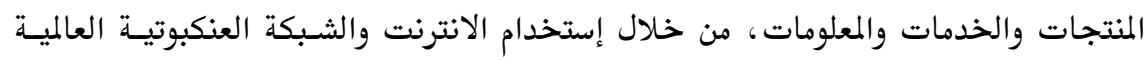

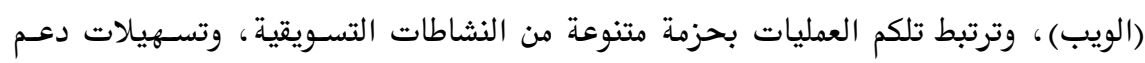
العمليات والخدمات ونظم المدفوعات الالكترونية وغيرها من النظم الالكترونية (36).

\section{ثانياً / فئات التجارة الالكترونية}

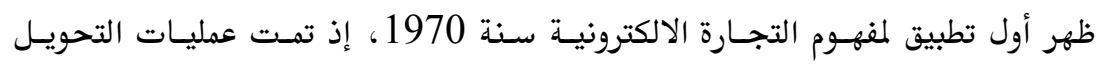

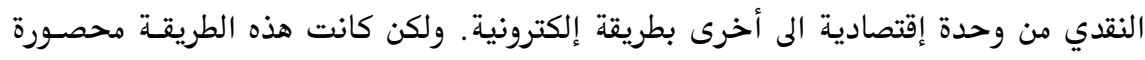

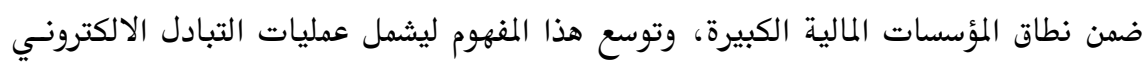

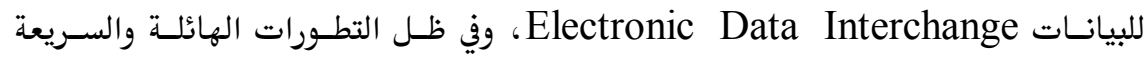
لنشاطات شبكات الحاسوب والبرمجيات، أخذت تظهر تطبيقات أخرى للتجارة الالكترونية

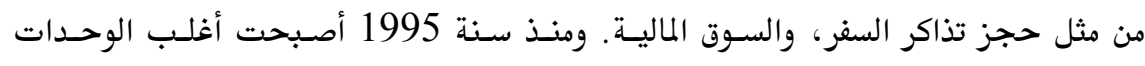

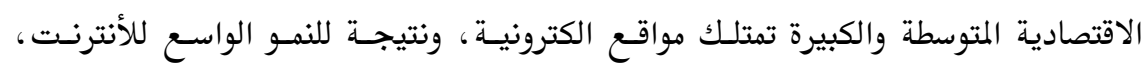

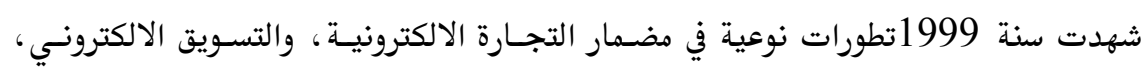

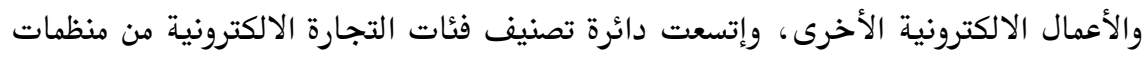

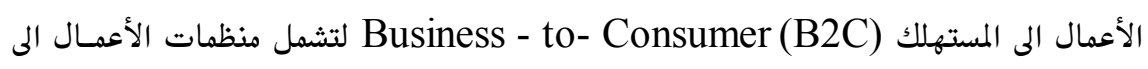

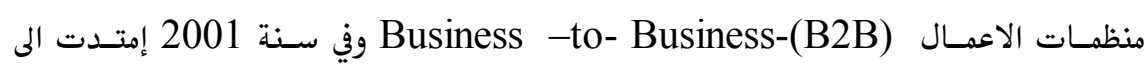

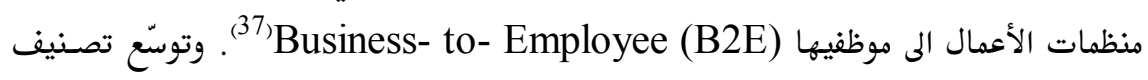
التجارة الالكترونية اليوم الى فئات عدة حسب طبيعة العلاقات القائمة بين الشركاء أو نوعية

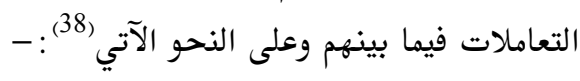




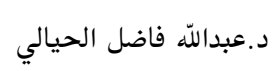
تطبيقات الذكاء الاقتصادي في التجارة..

- منظمات الأعمال الى منظمات الاعمال (B2B). - منظمات الأعمال الى المستهلك (B2C).

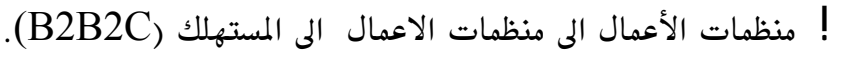
- المستهلك الى منظمات الاعمال (C2B). - المستهلك الى المستهلك (C2C).

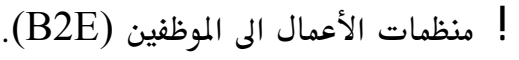

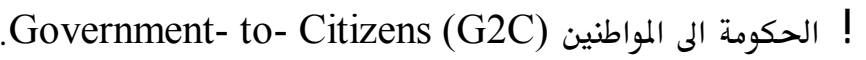

- التجارة عبر الهاتف المحمول Mobile-Commerce. - التجارة الالكترونية ضمن المنطقة الواحدة Intrabusiness EC. - تطبيقات المشاركة Peer- to- Peer Applications. - التجارة التحالفية Collaborative Commerce - E- Learning التعلم الالكترونية - عمليات الأعمال لدعم نشاطات البيع والشراء Business process that's support selling and purchasing activities.

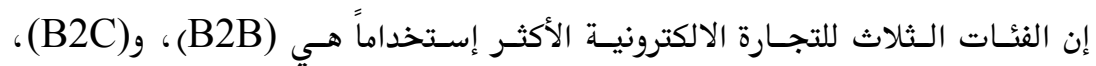

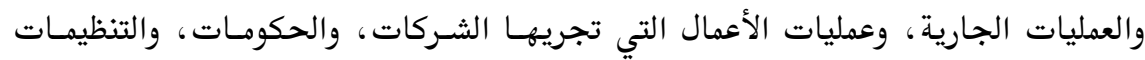

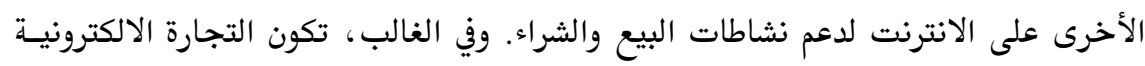

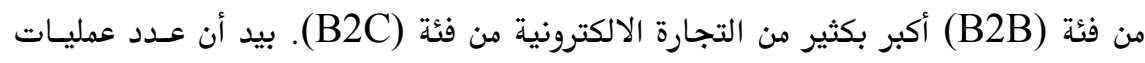

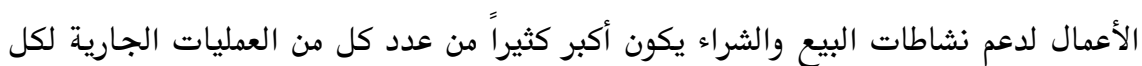
من فئة (B2B) وفئة (B2C) معاً (39). 


$$
\text { الجدول (1) }
$$

تطور المبيعات الفعلية والمتوقعة للتجارة الالكترونية للفئتين

(B2B) و (B2C) للمدة (B2C) (2007-1996)

\begin{tabular}{|c|c|c|}
\hline مبيعات (B2C) الفعلية & 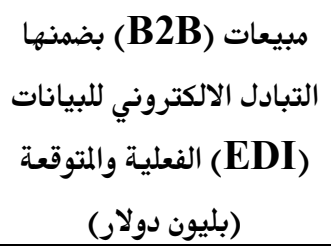 & السنوات \\
\hline أقل من 1 & 460 & 1996 \\
\hline 5 & 490 & 1997 \\
\hline 10 & 520 & 1998 \\
\hline 25 & 550 & 1999 \\
\hline 50 & 600 & 2000 \\
\hline 70 & 730 & 2001 \\
\hline 80 & 900 & 2002 \\
\hline 100 & 1600 & 2003 \\
\hline 130 & 2800 & 2004 \\
\hline 150 & 4100 & 2005 \\
\hline 190 & 5300 & 2006 \\
\hline 240 & 6800 & 2007 \\
\hline
\end{tabular}


د.عبدالله فاضل الحيالي تطبيقات الذكاء الاقتصادي في التجارة..

المصدر:- جاري شنايدر، التجارة الالكترونية، تعريب د. م. سرور علي إبراهيم سرور، دار المريخ للنشر، الرياض، المملكة العربية السعودية، 2008، ص 48.

يتبين من بيانات الجدول (1) أن مبيعات فئة (B2B) أكثر من مبيعات فئسة (B2C)، لأن عمليـات التبـادل الالكتروني للبيانـات (EDI) قائمسة وتعمـل بصـورة جيـدة منـذ سـنة 1995، فضلاً عن عمليات جارية بأكثر من (400) بليون دولار في السنة نفسها، مما جعـل

فئة (B2B) تنمو بشكل أكبر كونها تعتمد على قاعدة أكثر إتساعاً مقارنةً بفئة (B2C). ثالثاً/ الأهمية الاقتصادية للتجارة الالكترونية يمكن تقسيم الأهمية الاقتصادية للتجارة الالكترونية على مسـويين، الأول على مسـتى المستهلكين، والثاني على مستوى الوحدات الاقتصادية. أ) الأهمية الاقتصادية للتجارة الالكترونية على مستوى المستهلكين(40:) يمكن تلخيص ذلك بالنقاط الآتية : - - يمكسن للمسـتهـلك في نطـاق التجـارة الالكترونيـة الحصـول علـى المعلومـات المطلوبـة، والتسوّق، وإجراء الصفقات عبر شبكة الحاسوب، وهو في منزله أو مكتبـه بـدقائق عـدة بدلاً من الأيام والأسابيع مقارنة“ بما هي عليه الحال في ظل التجارة التقليدية.

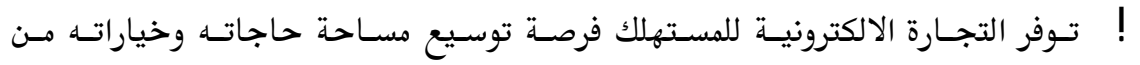
المنتجات والخدمات وبأسعار تنافسية رخيصة ومن المناشىء المحلية والعالمية كافة. - يستطيع المستهلك عبر التجارة الالكترونية الإتصال مـع نظرائسه وتبـادل الأفكـار والآراء والخبرات حول الموضوعات الاقتصادية المشتركة، فضلاً عن فرصة المشاركة في المـزادات الإفتراضية Virtual Auctions - يمكن للمستهلك الحصول على منتجـات وخـدمات بمواصـفات خاصـة بـه ، ويحسدّدها حسب حاجاته ورغباته. 
ب) الأهمية الاقتصادية للتجارة الالكترونية على مستوى الوحدات الاقتصادية: يمكن تلخيص ذلك بالنقاط الآتية(41): -

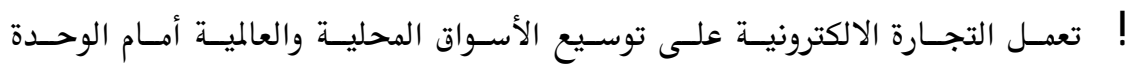

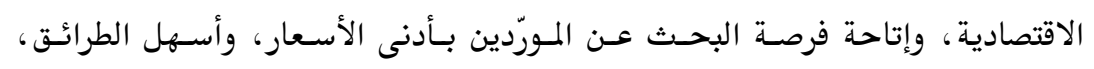

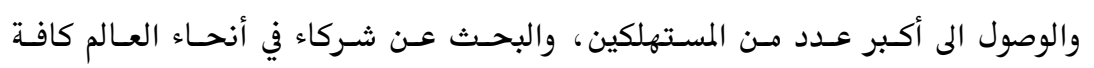

$$
\text { وبوسائل بيع سهلة وبكميات كثيرة. }
$$

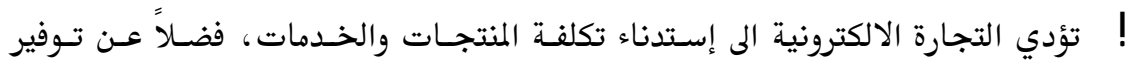

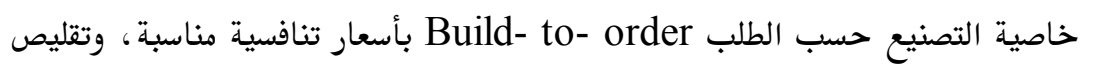
الحاجة الى إنشاء المخازن، وأحياناً الى إلغاءها.

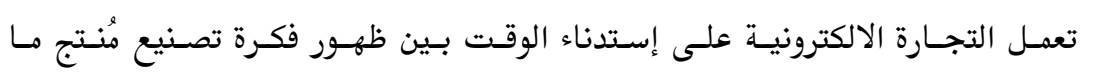

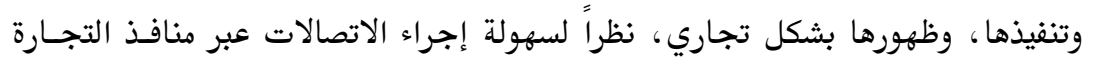
الالكترونية وخفض تكلفتها.

E- يمكن تعديل عرض المنتجات على الانترنت وأسعارها في أدلـة العـرض الالكترونيـة تكنة Catalogs

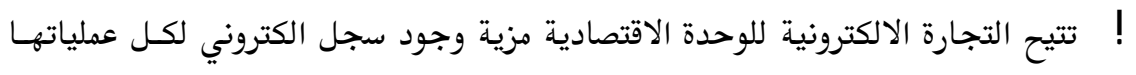

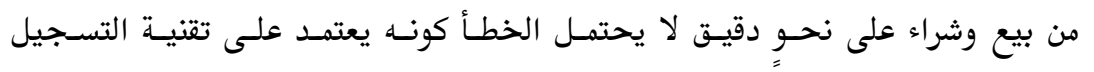

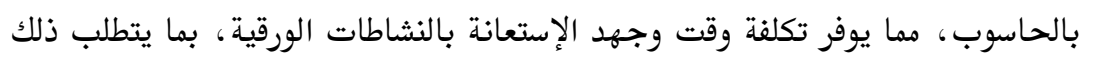

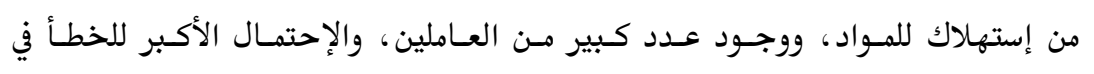
تسجيل البيانات (42). - ت تعمل التجارة الالكترونية على خفض تكلفة المعـاملات الماليـة بذحسو (80\% - 92\%) باستخدام النقد الالكتروني e-cash (حسبما أفادت إحدى الدراسات التطبيقية). فضلاً عن سرعة تدوير رؤوس الأموال، وزيادة الفرص أمام الوحدات الاقتصادية الصغيرة. 


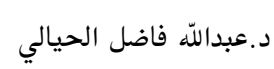
تطبيقات الذكاء الاقتصادي في التجارة..

يصف الاقتصاديــان كـريس فريمـان C.Freeman، وفرانسسكـو لوقـا F. Louca في

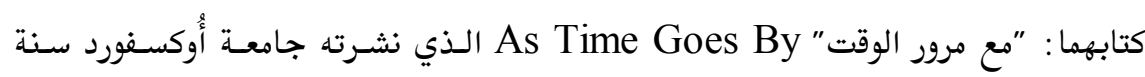

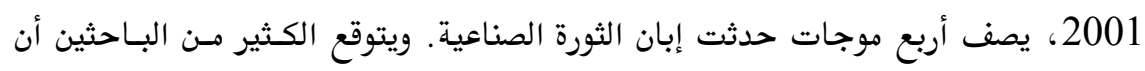

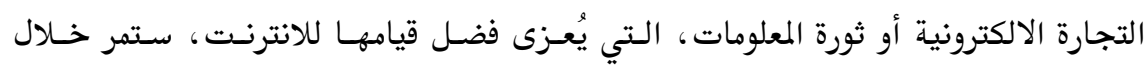

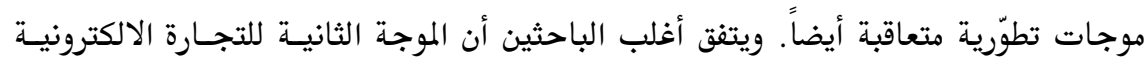
The second wave of EC

ولأجل تفحص أبرز خصائص المـوجتين الأولى والثانيـة للتجـارة الالكترونيـة، تم تصميم

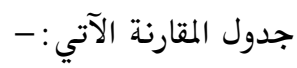
يلخص الجدول (2) السمات الرئيسة للموجتين الأولى والثانية للتجارة الالكترونية وعمل

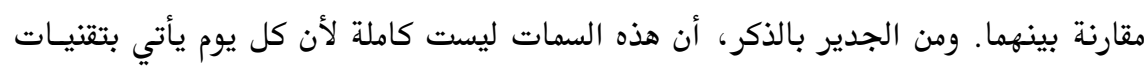

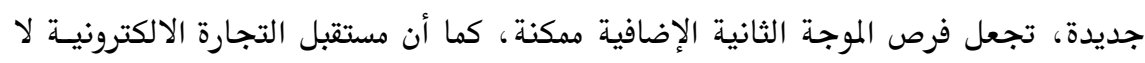

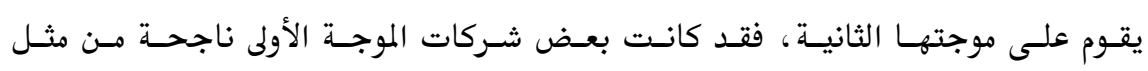

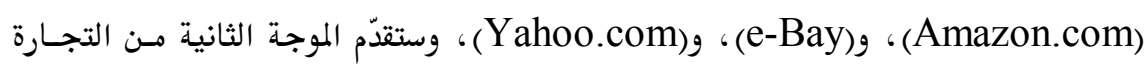
الالكترونية فرصاء جديدة لهذه الأعمال أيضاً (43). 
دراسات إقليمية 8 (24)

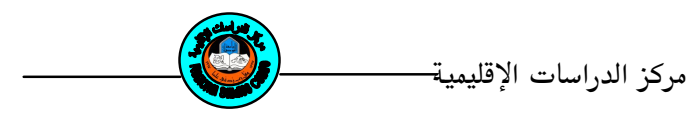

المبحث الثالث : - الذكاء الاقتصادي وأُنموذجات الأعمال في حقل التجارة الالكترونية

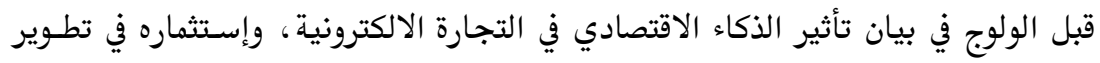

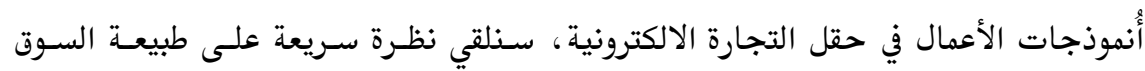

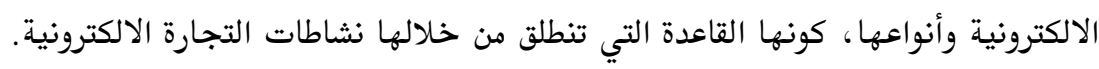
أولاًا طبيعة السوق الالكترونية تتصف السوق الالكترونية Marketspace بعسدد مـن السـمات أهمهـا تـوافر المنتجـات

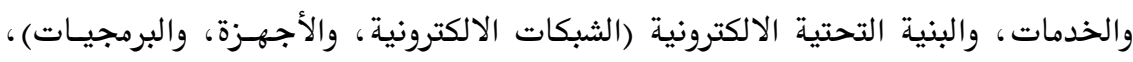


والبائع والمشتري سواء أكان البائع الأمامي Front end (مواقع البيع Seller’s portals،

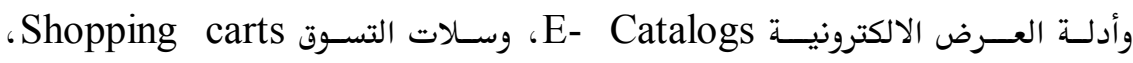

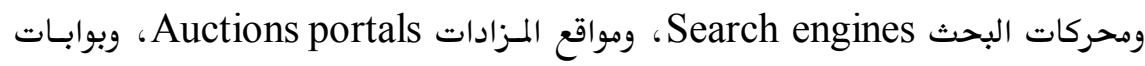

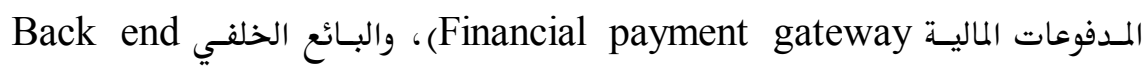

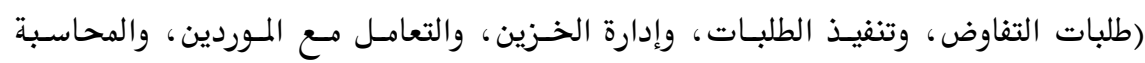

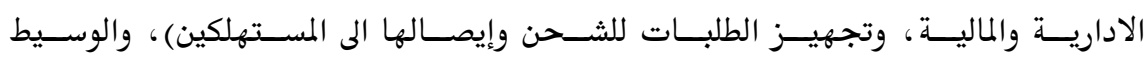
Intermediary Marketplace ويكمن الاختلاف الجوهري بين السوق الاعتياديـة Support services

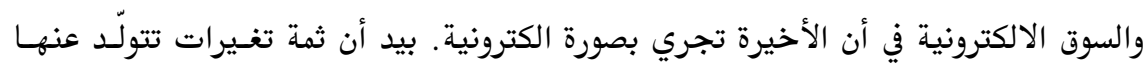

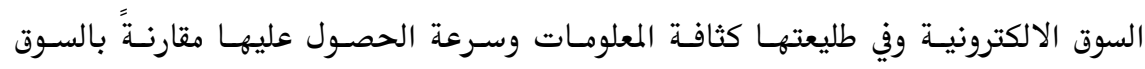

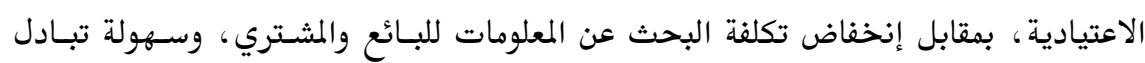

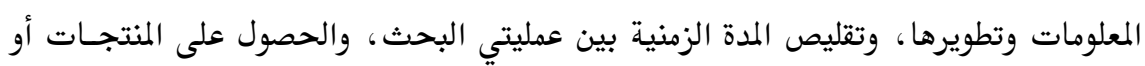

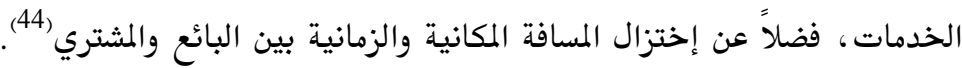

\section{ثانياً/ أنواع الأسواق في حقل التجارة الالكترونية}

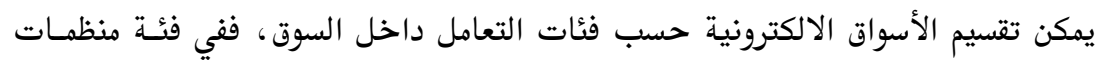

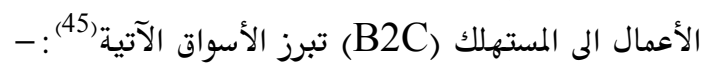

- المخازن الأمامية الالكترونية E- storefronts -

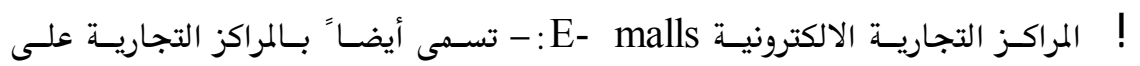

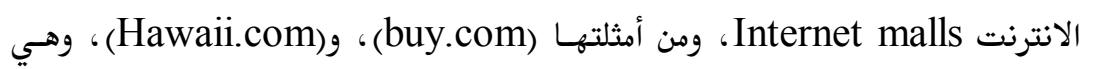

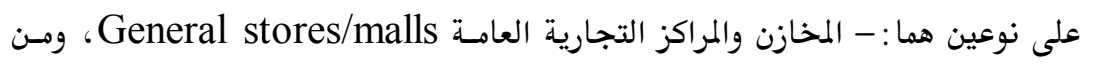

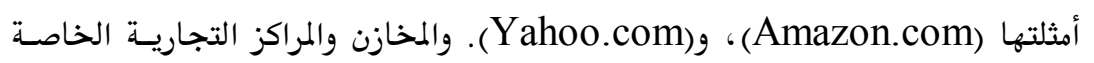

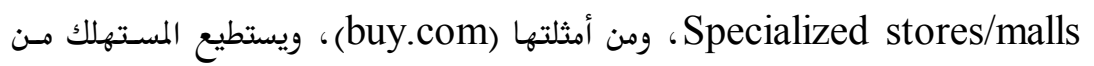
خلالها البحث عن أجهزة الحاسوب والمنتجات الرقمية حصراً. 


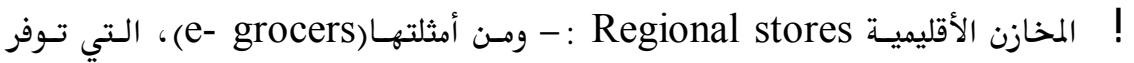

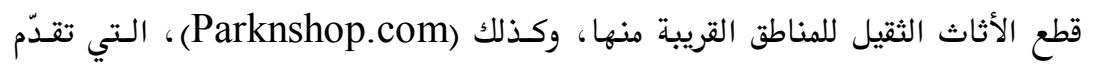
خدماتها حصرا" لمجتمع هونك كونك.

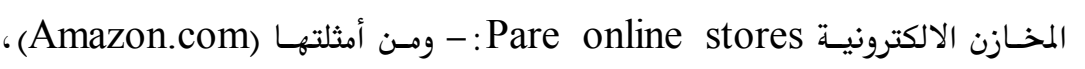

(Buy.com)و

أمـا فئسة منظمـات الأعمـال الى منظمـات الأعمـال (B2B) فـإن الأسـواق السـائدة فيهـا هي

- السـوق الالكترونيـة الخاصـة Private e-marketplace:- ينضسوي تحست لواءهـا

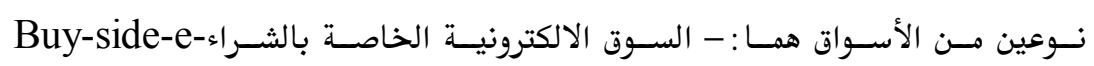
Sell-side-e-marketplace marketplace - السوق الالكترونية للتحالفات التجارية Consortia-e-marketplace. - السوق الالكترونية العامة السكونيه للحات النجات ثالثاً/تأثير الذكاء الاقتصادي في التجارة الالكترونية

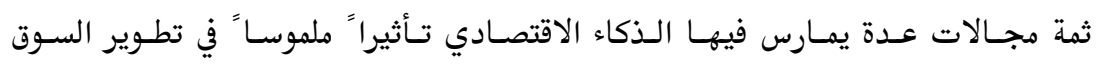
الالكترونية، وهي أساس عمل التجارة الالكترونية، ومن أبرز هذه المجالات ما يأتي : -

يعمل الذكاء الاقتصادي على زيادة الفرص المتاحة أمسام السـوق الالكترونيـة عـبر تنشـيط

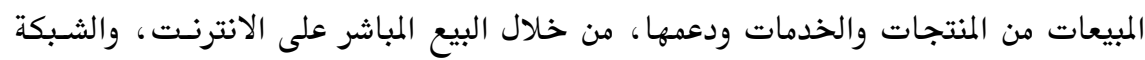
العنكبوتية العالمية (الويب).

- إنشاء قنوات بيع جديدة New sales channels تختلف أساليب الوصول الى المستهلكين باختلاف وسيلة الوصول. وقـد سـهّل الانترنست

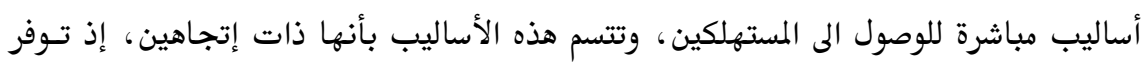

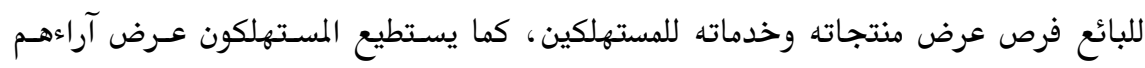


إزاء هذه المنتجات والخدمات، وتقبّل البـائع ردود فعـل المستهلكين. كمـا وفّرت التجـارة

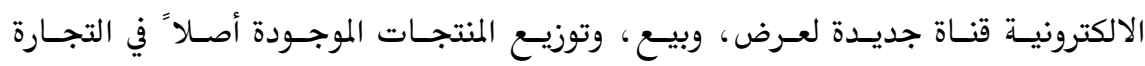

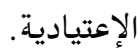

\section{Direct saving التوفير المباشر -}

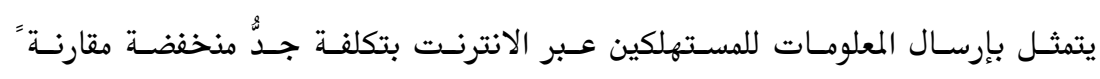

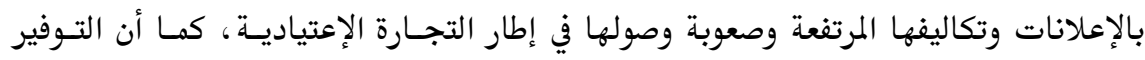

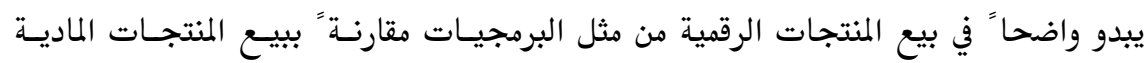
وتكلفة إيصالها.

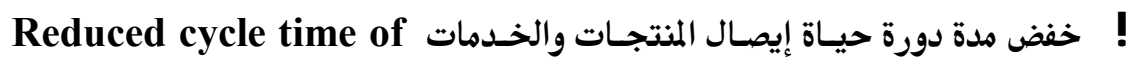
products $\&$ services

يمكن أن تصل المدة الزمنية لإيصال المنتجات والخدمات الرقميــة الى ثـواني عـدة، بينمـا

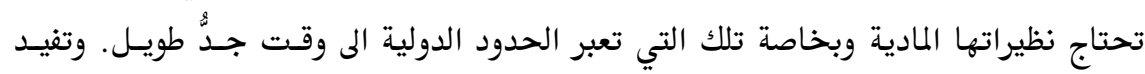

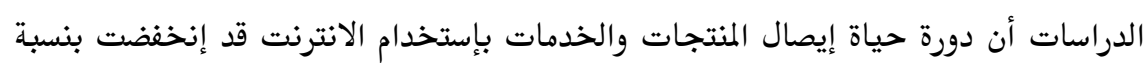
(47) (90\%)

\section{- تحسين خدمات المستهلكين Improved customer services}

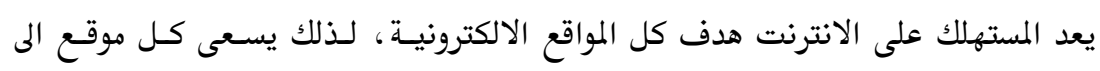

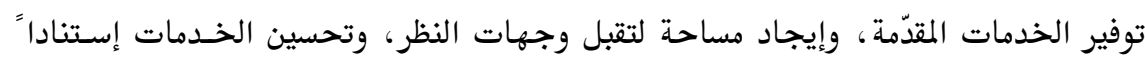

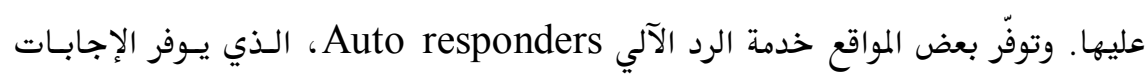

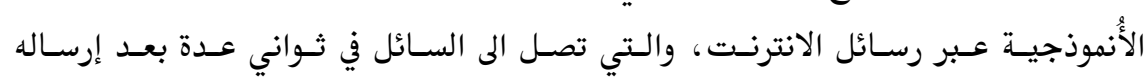


- إيجاد مذتجات وخدمات جديدة يتيح الذكاء الاقتصادي للوحدة الاقتصادية إنتاج منتجات وخدمات جدات جديسدة، فضـاً عـن

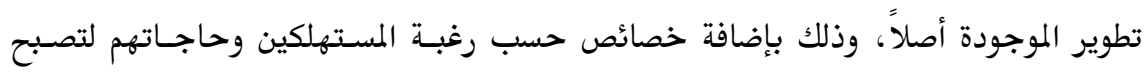

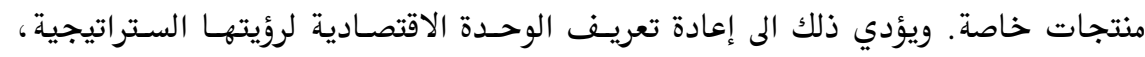

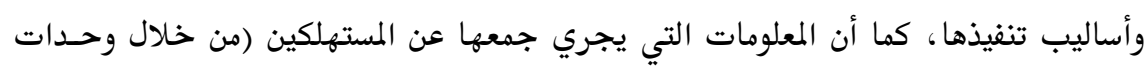

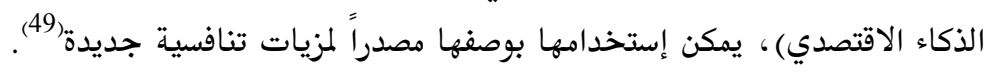

\section{Impacts on manufacturing التأثير في التصنيع -}

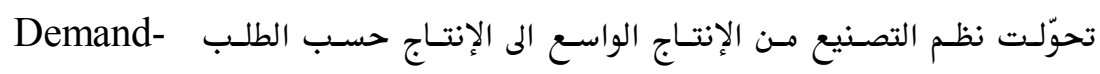
driven

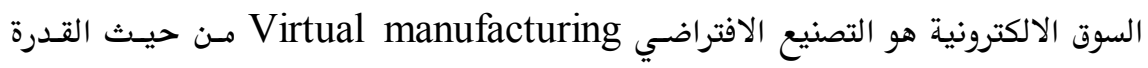

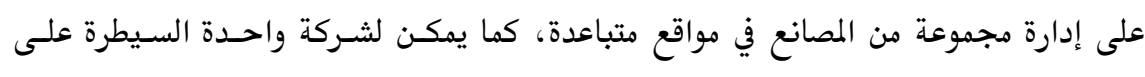

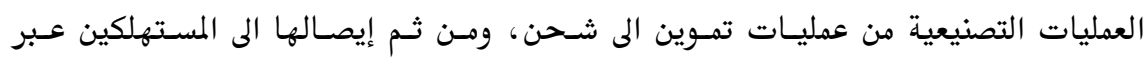

رابعا / إستثمار الذكاء الاقتصادي في تطوير أُنموذجات الأعمال في حقل التجارة الالكترونية أسفرت تطبيقات الذكاء الاقتصادي في حقل التجارة الالكترونية عـن أُنموذجــات جديسدة

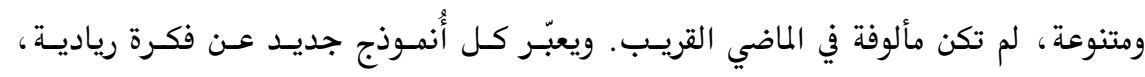

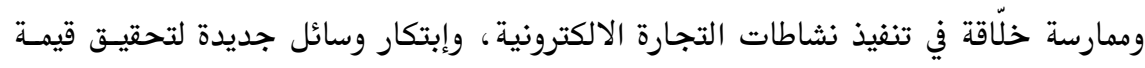

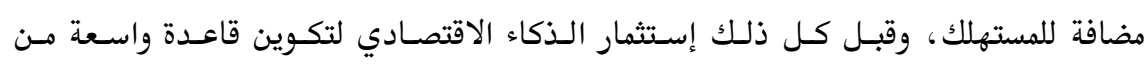
المستهلكين والمستفيدين في السوق الالكترونية على الصعيد العالمي.

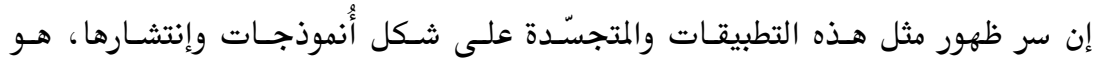

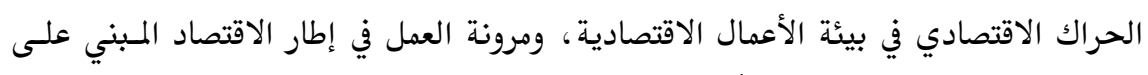

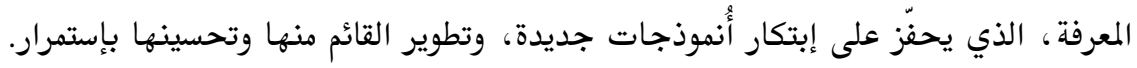




$$
\text { إن أبرز هذه الأنموذجات هي (52) :- }
$$

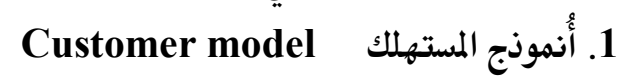

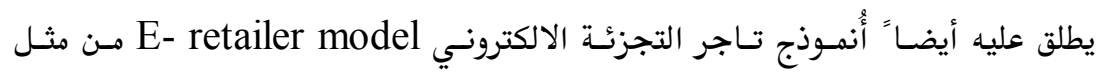

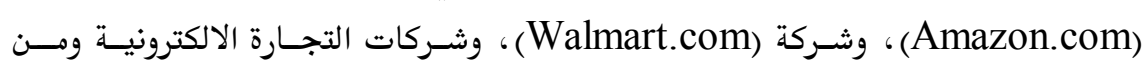

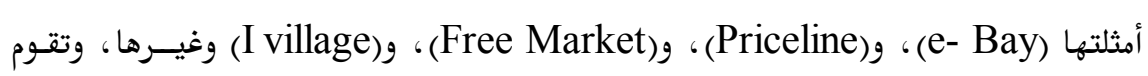
بنشاطـات البيع الفوري، وبأسعار محددة، أو بأسعار قابلة للتفاوض.

\section{2. أُنموذج وسيط المعلومات الالكتروني E- informediary model}

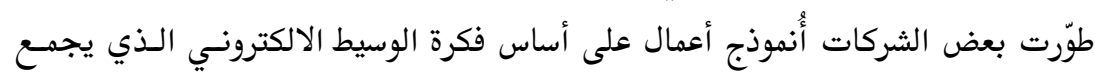

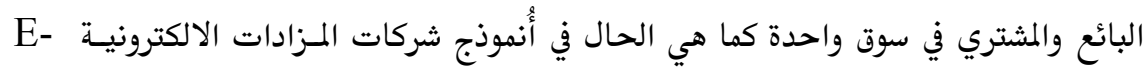
auctions

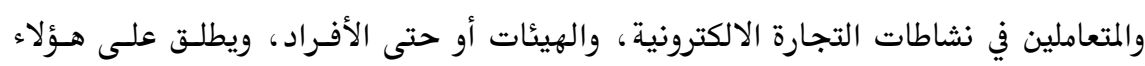

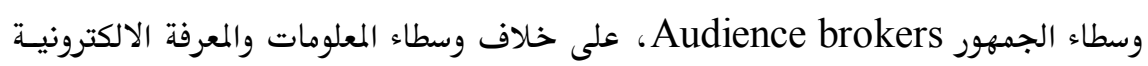
الذين يعملون في مواقعهم على الشبكة العنكبوتية العالمية (الويب)، من خلال مصارف كبيرة

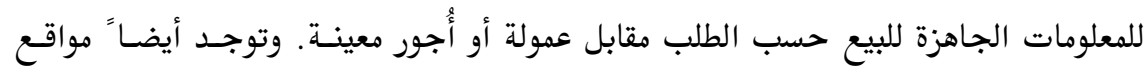

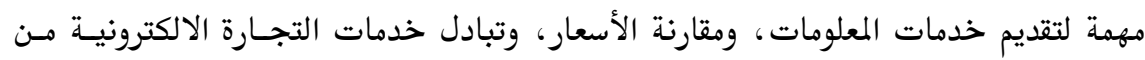

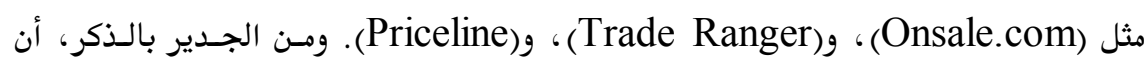

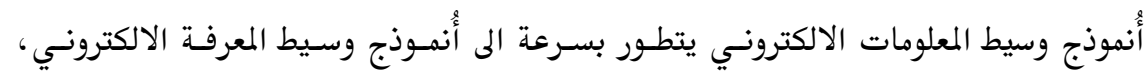

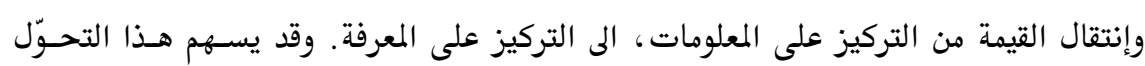

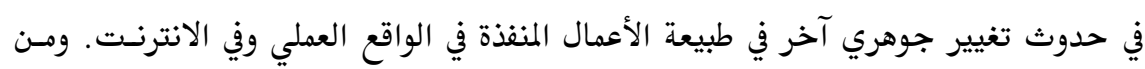

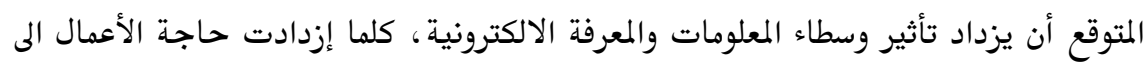

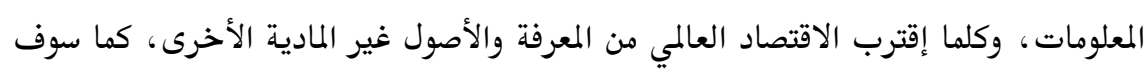

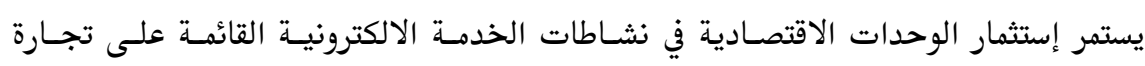

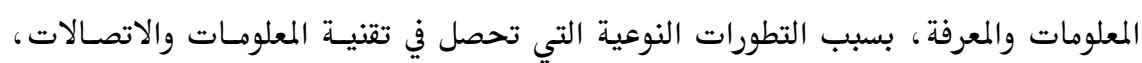

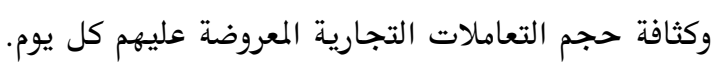


3. أُنموذجات مجهزي تسهيلات البنية التحتية الالكترونية

\section{E- infrastructure facilities providers models}

قامـت الثـركات المجهـزة لتسـهيلات وخـدمات البنيـة التحتيـة للأعمـال الالكترونيـة

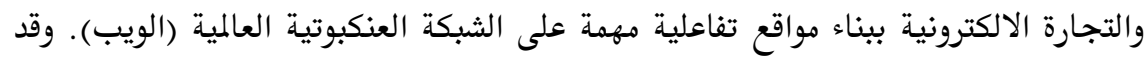

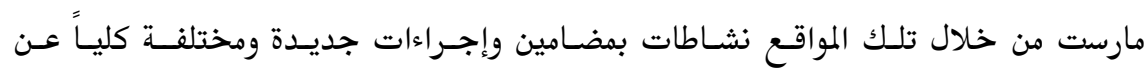

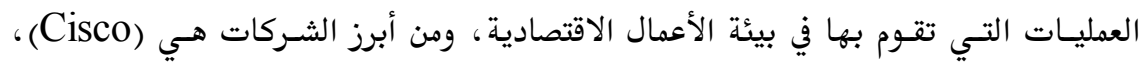

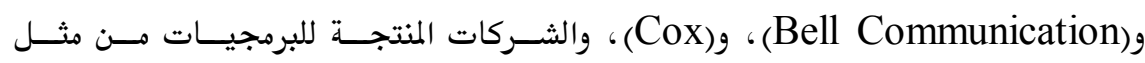

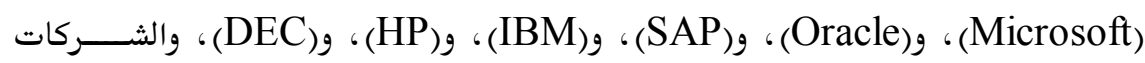

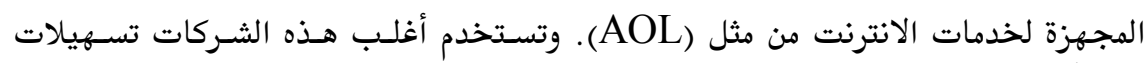

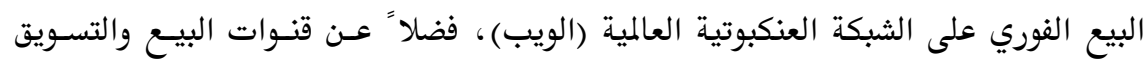
التقليدية.

$$
\text { 4. أُنموذج مجهِّز محتوى المعلومات الالكتروني }
$$

\section{E- information content provider model}

يشير هذا الأُموذج الى كل أشكال تجهيز محتوى المعلومات من نصوص وأخبـار رقميـة،

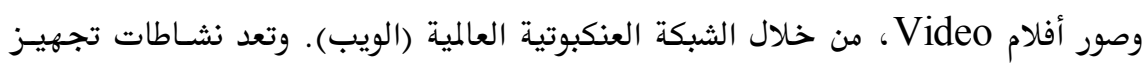
محتـوى المعلومـات الالكترونسي ثـاني أكسبر مصدر للإيـرادات في التجــارة الالكترونيــة لفئسة

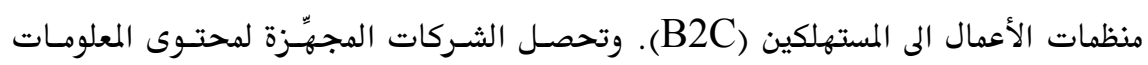

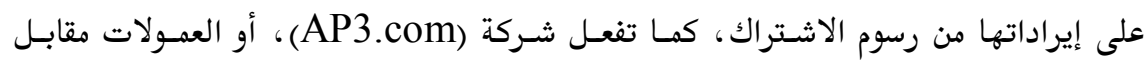

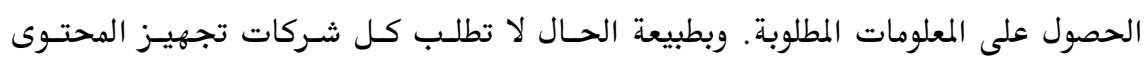

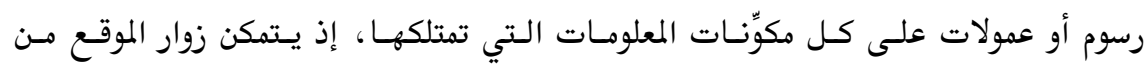

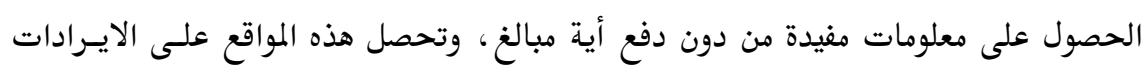

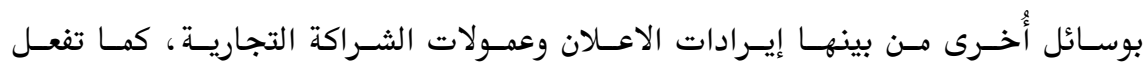

(Thestandard.com)، (CIO.com) 
5ortal models أُنموذجات مواقع النوافذ الكبرى هي مواقع مهمة على الشبكة العنكبوتية العالمية (الويب)، تُستخدم بحسبانها محركات

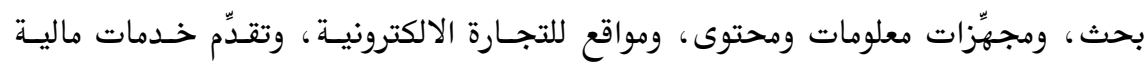

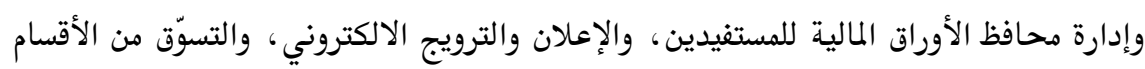

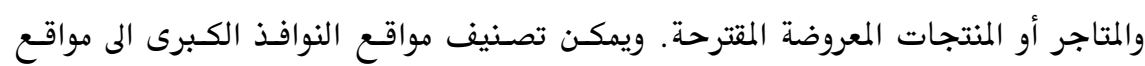

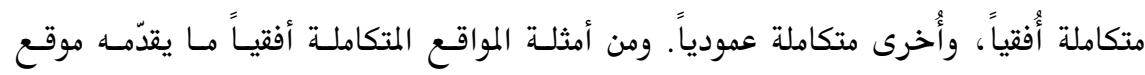

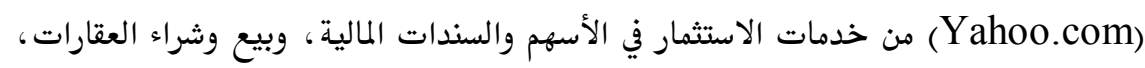

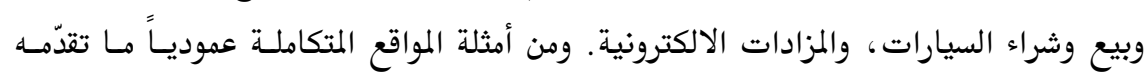

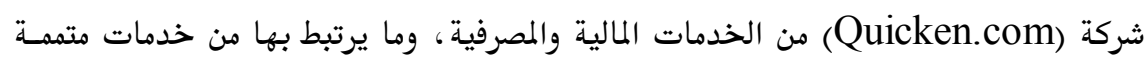

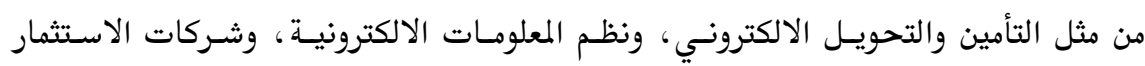

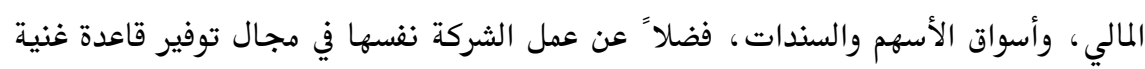
من المعلومات للمستهلكين والمستفيدين الآخرين.

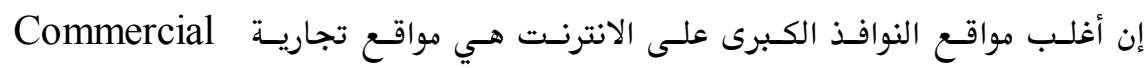
portals

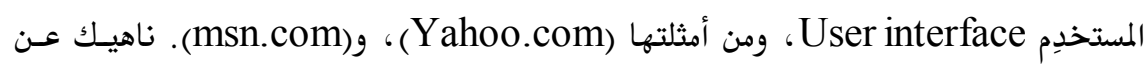
مواقع التعاونCorporate portals، التي توفر معلومات غزيرة وغنية بالمحتويـات لعـدد

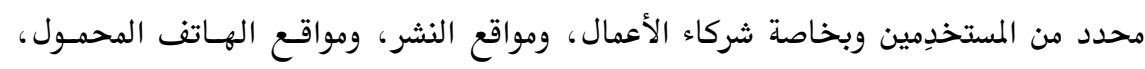

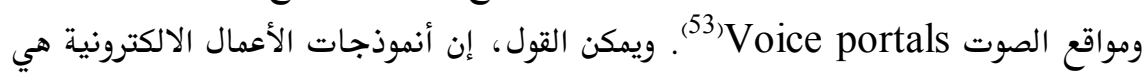

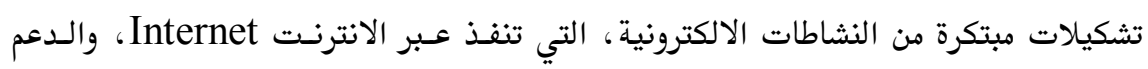

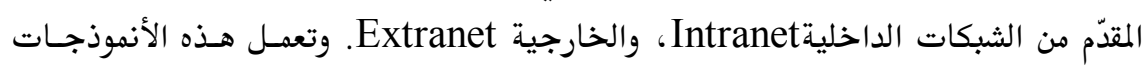

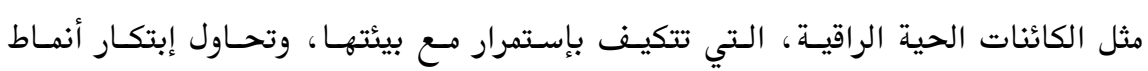
وأشكال جديدة من العمل المثمر ذي القيمة المضافة(54). الاستنتاجات والتوصيات 
تمكن البحث من الخلوص الى الاستنتاجات الآتية :-

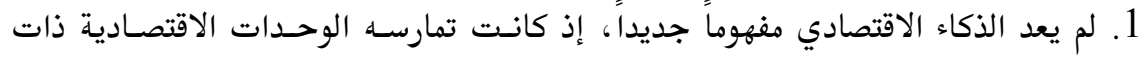

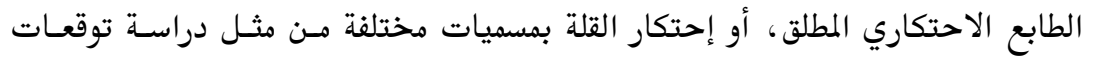
المنتجين، وتنبؤات الطلب، ودراسة واقع السوق الحالية والمستقبلية وغيرها تداركاً منها

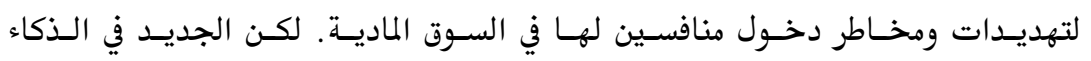

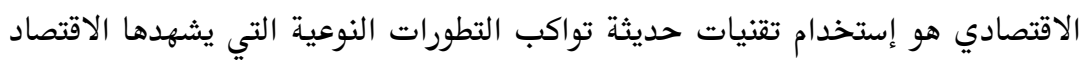

$$
\text { المبني على المعرفة. }
$$

2. يعد الذكاء الاقتصادي أقوى وسيلة معرفـة للوحسدة الاقتصـادية، عـبر إستغلالها لفيض الفيض

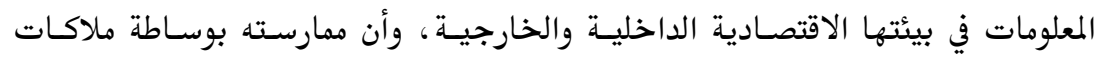
متخصصة يعد عامل تطوير للمعلومات وتدفقها بما يؤدي الى تحقيق قيمة مضـافة جديـدة لها وزيادة قدرتها التنافسية في السوق.

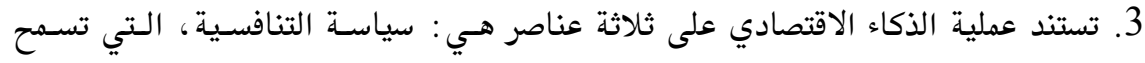

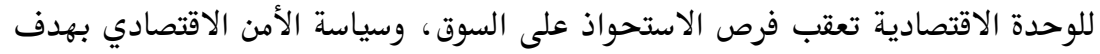

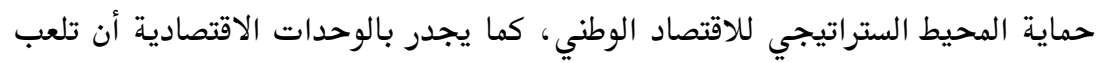

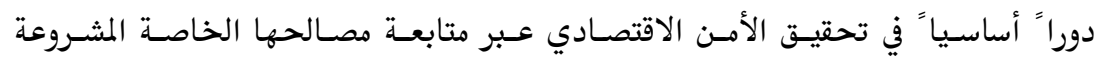

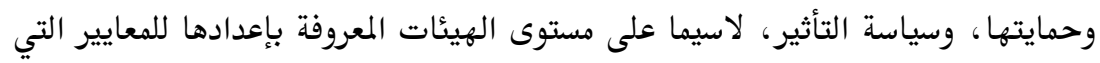
تدير الحياة الاقتصادية. 4. لم يعد التحدي اليوم يتمثل في توفير المعلومات حسب، واندانه وانما في سـرعة الوصول إليهـا،

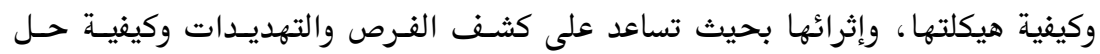

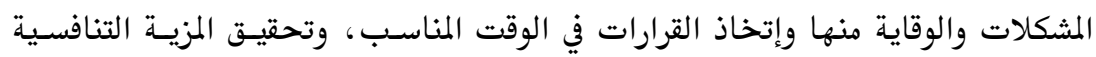
للوحدة الاقتصادية.

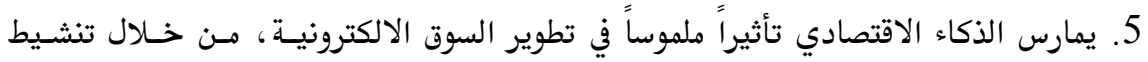

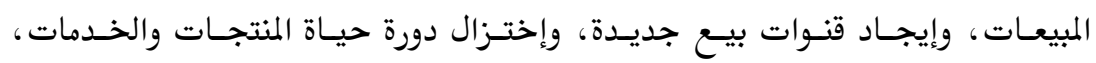

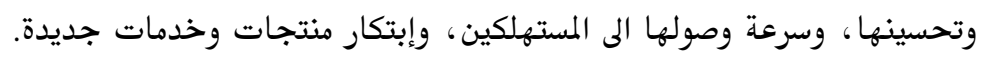
6. إن أُنموذجات الأعمال الالكترونية هي تشكيلات مبتكرة من النشاطات الالكترونيسة التني

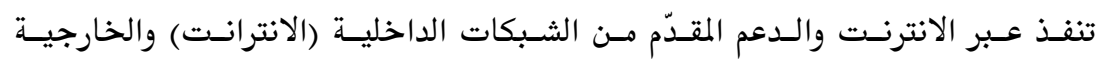




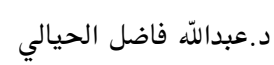
تطبيقات الذكاء الاقتصادي في التجارة..

(الاكسترانت) للوحدة الاقتصادية، وتعمل هذه الأنموذجات مثل الكائنات الحية الراقيـة

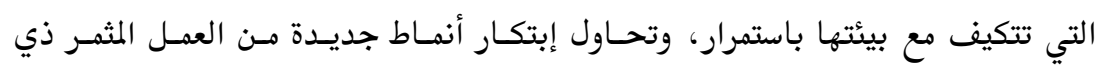

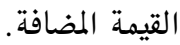

7. تحتاج عملية الذكاء الاقتصادي الى تعلّم تقنيات جديدة، وأن نجـاح إقامـة هـذه العمليـة يتوقف على مدى التطبيق العملي والذكي لهذه التقنيات. ثانياً /التوصيات

$$
\text { ينتهي البحث بتقديم التوصيات الآتية : - }
$$

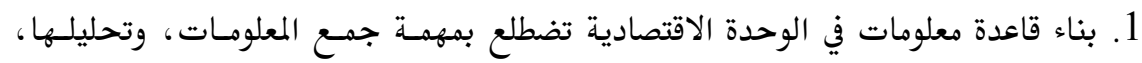
وأن يكون مدير إدارتها عضواً في مجلس إدارة الوحدة الاقتصادية.

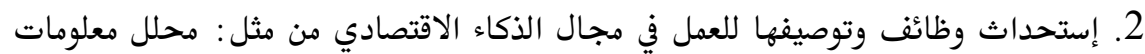

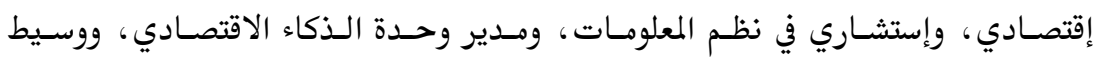
معلومات، ومتخصص في اليقظة الاقتصادية أو التجارية أو التنافسية.

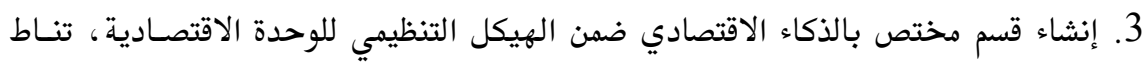

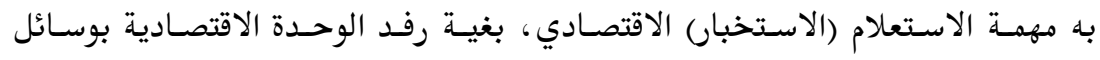

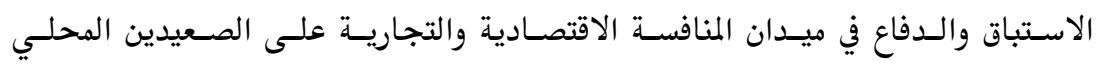
والدولي. 4. إستحداث هيئة عامة للذكاء الاقتصادي على مستوى الاقتصـاد الـوطني، تتـولى إنضـاج

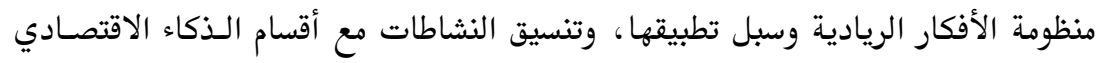
في الوحدات الاقتصادية. 5. تأهيل ملاكات متخصصة في الذكاء الاقتصادي على مستوى القطاعين العام والخاص، من الونصاديه

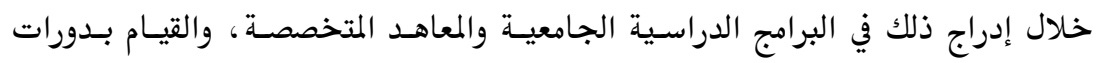

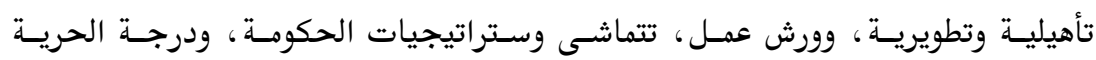

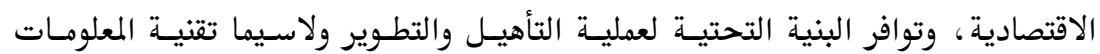

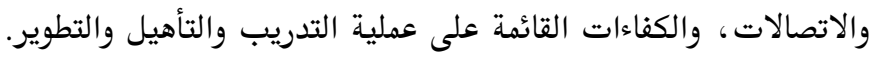

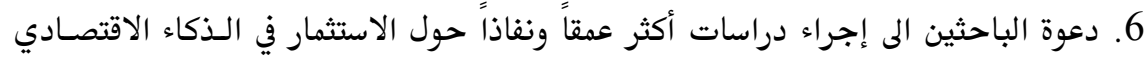

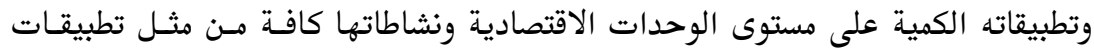




$$
\begin{aligned}
& \text { مركز الدراسات الإقليمية- (24) } \\
& \text { الذكاء الاقتصادي في مجـال تطوير منتجـات جديـدة، وإيجـاد منافـذ تسـويق جديـدة، } \\
& \text { وإستحداث طرائق إنتاج جديدة، وإبتكار مزيات تنافسية وغيرها. }
\end{aligned}
$$

\title{
Economic Intelligence Applications In Electronic Commerce In Frame of Knowledge- Based Economy
}

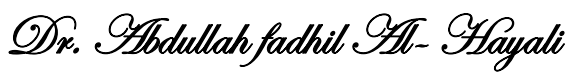 \\ Qlecturer / Head of Exonomic and Pocial Pludies Department \\ Regional Qhudies Genter Olniversily of Shosul
}

\begin{abstract}
Economic Intelligence has become a new source for economic units in getting competitive advantage and achieving profit through functioning technologies to get the accurate information with high quality value and reliability. Treatment and diffusion of information in order to reduce uncertainty in the process of making strategic decisions.

One of the most significant fields in applying economic intelligence is the field of electronic commerce for the marketspace are considered a source for value added and a fertile place in getting information and using these information in rational way.

Also knowing the type of competitors, the essence of new digital and physical services and products that can be marketing, and searching for new investments, knowing the sort of present and propable customers, their needs and wants, knowing more profitable services and products. Also the competent planning of advertising campaigns in order to increase the opportunities of promotion the sales and providing new channels of sales, supplying services and products, improving and distributing them, and decreasing cycle time in reaching these products \& services to the consumers.

In other words, the economic intelligence applications in the field of electronic commerce is considered a fertile field to achieve more
\end{abstract}


revenues in the light of knowledge -based economy, which is considered the most expanded, competitive and profitale economy.

$$
\text { هوامش البحث ومصادره }
$$

(1) $<<$ http://www.ces.fr/rapport/rapsec/R5052710.pdf $>>$

(2) V.pirttimaki \& M.Hannula, "Finnish Experience of Business Intelligence Practices", International Conference on Business Intelligence,Oct., 16, 2003, Vitoria, Museo, Artium, Francia, 2003. In:

M. peltoniemi \& E-Vuori, Competitive Intelligence and co- evolution within an organisation population, $\mathbf{P} .2$.

$<<$ http://www.tut.fi $>>$.

(3) -H. Chen, M.Chau and D. Zeng, "CI spider: a tool for competitive intelligence on the web", Decision Support Systems, vol.34, 2002, p.2.

-J.R. Groom \& F.R. David, "Competitive intelligence activity among small firms", SAM Advanced Management Journal, Winter 2001, p.13.

(4) H.Chen, M. Chau and D.Zeng, op.cit., p.1.

(5) Ibid., p.2.

(6) M.C. Attaway, Competitive Intelligence, Internal Auditor, Dec. 1999, p.50.

(7) Babajide Afolabi \& Odile Thiery, "Business intelligence systems and user's parameters: an application to a document's database".

$<<$ http://www.babajide.afolabi@loria.fr,Odile. thiery@, loria.fr $>>$.

(8) $<<$ http://www. Medefparis. fr/Livre Blanc.pdf $>>$.

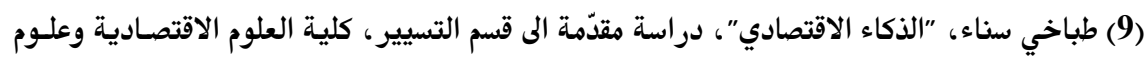

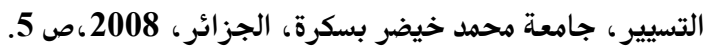

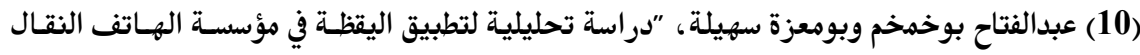

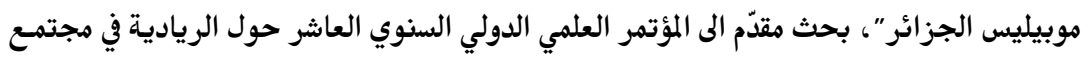


المعرفة الذي أقامته جامعة الزيتونــة الأردنيـة الخاصـة، كليـة الاقتصـاد والعلوم الاداريـة للمـدة

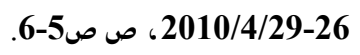

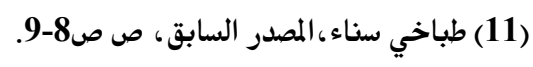

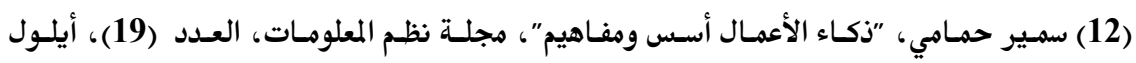

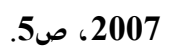

$<<$ http://www.infomag.news.sy $>>$

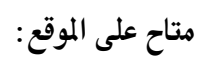

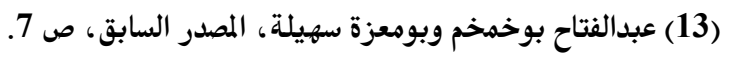

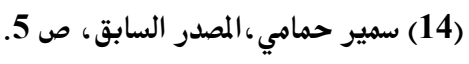

(15) طباخي سناء، المصدر السابق، ص ص 9.

(16) $<<$ http://www.espionageinfo.com/Ec-Ep/Economic- Intelligence.html >> (17) طباخي سناء، المصدر السابق، ص 10.

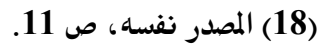

$$
\text { (20) المكان نفسه. }
$$

(21) د. عبدالرزاق خليل ود. أحلام بوعبدلي، "الذكاء الاقتصـادي في خدمسة هنظمسة الأعمال"، دراسـة

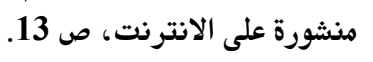

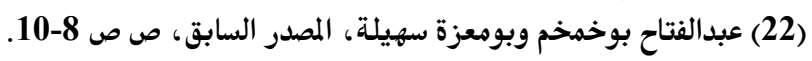

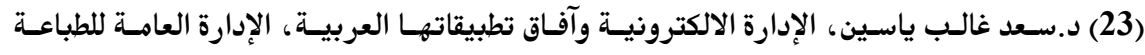

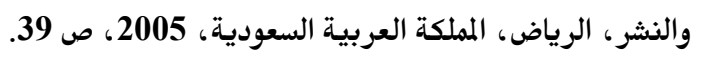

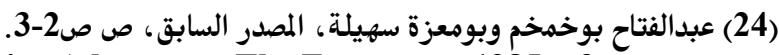

(25) M.Porter, Competitive Advantage, The Free press, 1985,p.3.

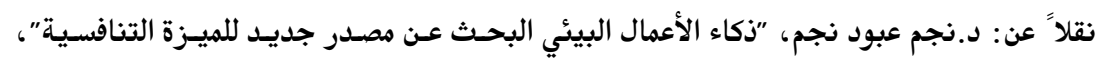

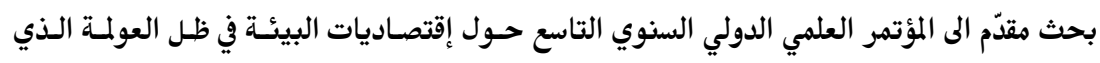

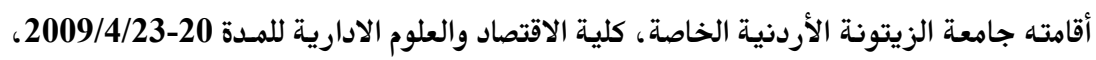

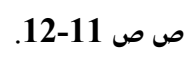

(26) W.E. Stead et al, Sustainable Strategic Management, M.E. Sharpe, Armonk, 2004, p.27 and pp.45-46.

$$
\text { (27) المقدرً عن: د.نجم عبود نجم، المصدر السابق، ص ص12-11-12. }
$$

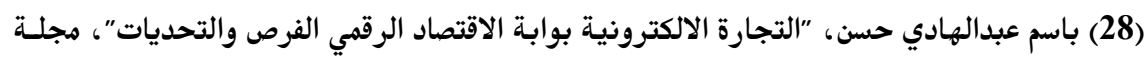

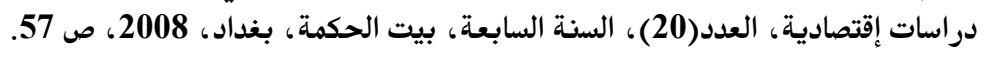




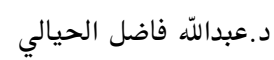

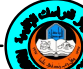

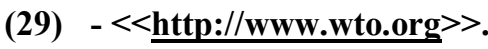

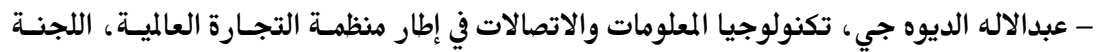

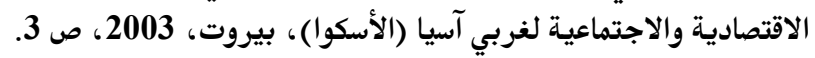

(30) $<<$ http;//www.worldbank.org $>>$.

(31) $<<$ http://www.oecd.org $>>$.

(32) أحمد سفر، العمل المصرفي الالكتروني في البلدان العربية، المؤسسـة الحديثـة للكتـاب، طـرابلس،

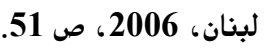

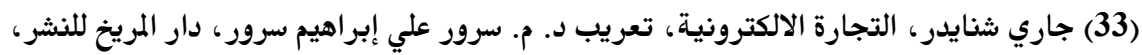

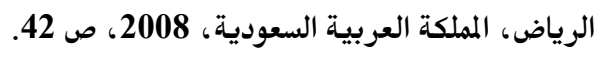

(34) Greenstein Marilyn \& Feinman Tood, Electronic Commerce: Security,

Risk, Management and Control, Irwin. McGraw- Hill, Boston, 2001, p.2.

(35) E.Turban, D.king, J.Lee and D. Viehland, Electronic Commerce: A managerial Perspective, New Jersey: Pearson Prentice Hall, 2004.

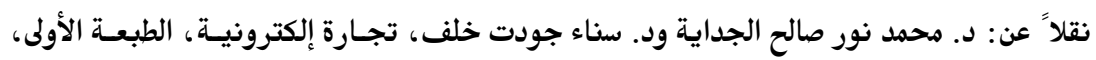

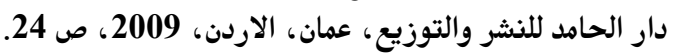

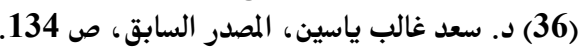

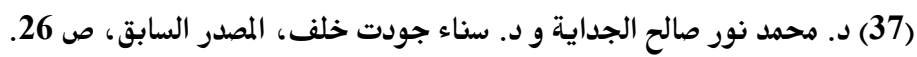

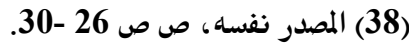

(39) جاري شنايدر، المصدر السابق، ص ص ص صد 42 -43 -43.

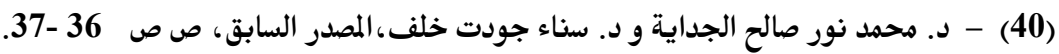

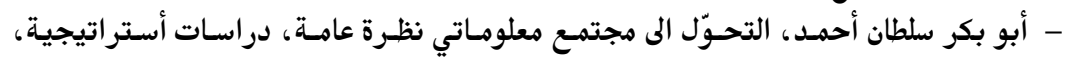

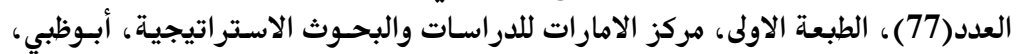

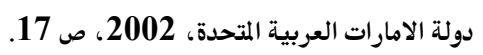

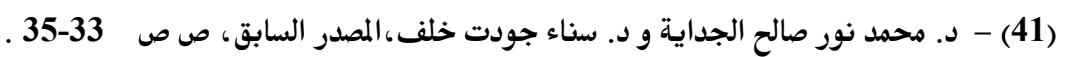

- M.T. Matt,'The Economics of e- cash", IEEE Spectrum, vol. 34, no. 2, N.Y,

Feb. 1997, pp. 69- 73.

- S. H.Bakry, "e- Business Trends", Nov. 2000.

>>http://www.insightview.com/ebizschool/courses/ebtrn.pdf $>$ متاح على الموقع:

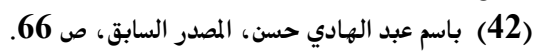

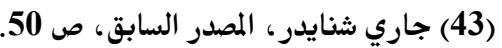

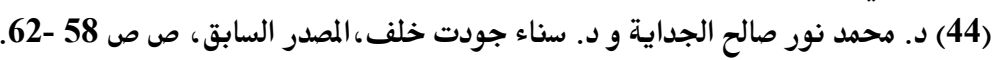

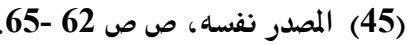

(46) (المصدر نفسه، ص ص صنسه ص 66 -67 -65. 
دراسات إقليمية 8 (24)

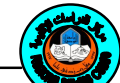

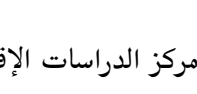

$$
\begin{aligned}
& \text { (47) المصدر نفسه، ص } 99 .
\end{aligned}
$$

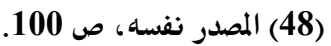

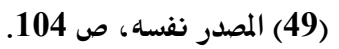

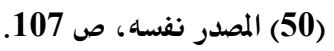

$$
\begin{aligned}
& \text { (51) د. سعد غالب ياسين، المصدر السابق، ص } 174 \text { صالمد } 174 .
\end{aligned}
$$

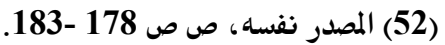

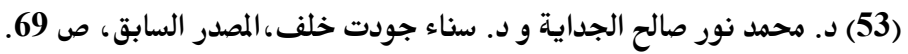

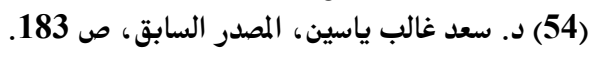

